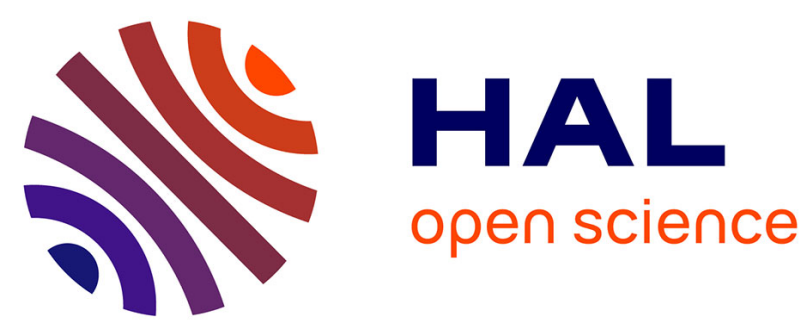

\title{
Immiscible two-phase Darcy flow model accounting for vanishing and discontinuous capillary pressures: application to the flow in fractured porous media
}

Konstantin Brenner, Mayya Groza, Laurent Jeannin, Roland Masson, Jeanne Pellerin

\section{To cite this version:}

Konstantin Brenner, Mayya Groza, Laurent Jeannin, Roland Masson, Jeanne Pellerin. Immiscible twophase Darcy flow model accounting for vanishing and discontinuous capillary pressures: application to the flow in fractured porous media. Computational Geosciences, 2017, 10.1007/s10596-017-9675-7 . hal-01338512v3

\section{HAL Id: hal-01338512 \\ https://hal.science/hal-01338512v3}

Submitted on 15 Sep 2017

HAL is a multi-disciplinary open access archive for the deposit and dissemination of scientific research documents, whether they are published or not. The documents may come from teaching and research institutions in France or abroad, or from public or private research centers.
L'archive ouverte pluridisciplinaire HAL, est destinée au dépôt et à la diffusion de documents scientifiques de niveau recherche, publiés ou non, émanant des établissements d'enseignement et de recherche français ou étrangers, des laboratoires publics ou privés. 
Noname manuscript No.

(will be inserted by the editor)

\section{Immiscible two-phase Darcy flow model accounting for vanishing and discontinuous capillary pressures: application to the flow in fractured porous media}

\author{
Konstantin Brenner ${ }^{1,2}$, \\ Mayya Groza ${ }^{1,2}$, \\ Laurent Jeannin ${ }^{3}$, \\ Roland Masson ${ }^{1,2}$ and \\ Jeanne Pellerin ${ }^{4}$ \\ 1 Laboratoire de \\ Mathématiques J.A. \\ Dieudonné, Université \\ Côte d'Azur, Nice \\ 2 Team Coffee INRIA \\ Sophia Antipolis \\ Méditerranée, Valbonne \\ ${ }^{3}$ Expertise Centre - Sub \\ Surface Department, \\ Storengy, Bois Colombes \\ ${ }^{4}$ Weierstrass Institute, \\ Berlin
}

\begin{abstract}
Fully implicit time-space discretizations applied to the two-phase Darcy flow problem lead to the systems of nonlinear equations, which are traditionally solved by some variant of Newton's method. The efficiency of the resulting algorithms heavily depends on the choice of the primary unknowns since Newton's method is not invariant with respect to a nonlinear change of variable. In this regard the role of capillary pressure/saturation relation is paramount because the choice of primary unknowns is restricted by its shape. We propose an elegant mathematical framework for twophase flow in heterogeneous porous media resulting in a family of formulations, which apply to general monotone capillary pressure/saturation relations and handle the saturation jumps at rocktype interfaces. The presented approach is applied to the hybrid dimensional model of two phase water-gas Darcy flow in fractured porous media for which the fractures are modeled as in-
\end{abstract}

Address(es) of author(s) should be given terfaces of co-dimension one. The problem is discretized using an extension of Vertex Approximate Gradient scheme. As for the phase pressure formulation, the discrete model requires only two unknowns by degree of freedom.

\section{Nomenclature}

\section{Continuous problem}

$\Omega \quad$ Matrix domain.

$\left(\Gamma_{i}\right)_{i \in I} \quad$ The set of individual fractures.

$\Gamma \quad$ The fracture network.

$m, f \quad$ The subscripts associated to the quantities defined on the matrix domain, and on the fracture network respectively.

$\phi_{m}, \phi_{f} \quad$ Porosity.

$d_{f} \quad$ Fracture width.

$\Lambda_{m} \quad$ Matrix permeability tensor.

$\Lambda_{f} \quad$ Fracture tangential permeability tensor.

$k_{r, m}^{\alpha}, k_{r, f}^{\alpha} \quad$ Relative permeability of the phase $\alpha$.

$\mathbf{q}_{m}^{\alpha} \quad$ Matrix Darcy velocity of the phase $\alpha$.

$\mathbf{q}_{f}^{\alpha} \quad$ Fracture tangential Darcy velocity of the phase $\alpha$ integrated other the fracture width.

$\rho^{\alpha} \quad$ Density of the phase $\alpha$.

$\mu^{\alpha} \quad$ Viscosity of the phase $\alpha$.

$s^{\alpha} \quad$ Saturation of the phase $\alpha$.

$u^{\alpha} \quad$ Pressure of the phase $\alpha$.

$p \quad$ Capillary pressure $p=u^{g}-u^{w}$.

$g, w \quad$ The superscripts $g$ for gas and $w$ for water.

$S_{m}^{g}, S_{f}^{g} \quad$ Inverse of the capillary pressure graph.

$\left(\Omega_{j}\right)_{j \in J_{m}} \quad$ The set of rocktypes within the matrix domain.

$\left(\Upsilon_{j}\right)_{j \in J_{f}} \quad$ The set of rocktypes within with the fracture network.

$\left(S_{m, j}^{g}\right)_{j \in J_{m}}$ The set of inverse capillary pressure graphs associated with matrix rocktypes.

$\left(S_{f, j}^{g}\right)_{j \in J_{f}}$ The set of inverse capillary pressure graphs associated with fracture network rocktypes.

g Gravity vector given by $\mathbf{g}=g \nabla z$.

\section{VAG discretization}

$\mathcal{M} \quad$ The set of polyhedral cells of the mesh.

$\mathcal{F}, \mathcal{F}_{\Gamma}, \mathcal{F}_{K}$ The set of polygonal faces, its subset of faces lying on the fracture network and the subset of faces lying on the boundary of the cell $K \in \mathcal{M}$.

$\mathcal{V}, \mathcal{V}_{\sigma} \quad$ The set of mesh nodes and its subset of nodes lying on the boundary of the face $\sigma$.

$\mathcal{V}_{\text {ext }}, \mathcal{V}_{\text {int }}$ The subsets of "external" and "internal" nodes of the mesh.

$\Xi_{K} \quad$ The set of degrees of freedom associated with the boundary of the cell $K \in \mathcal{M}$. 


\begin{tabular}{|c|c|}
\hline$\phi_{K}$ & $\begin{array}{l}\text { Porous volume associated to the cell } K \in \\
\mathcal{M}\end{array}$ \\
\hline$\phi_{K, \nu}$ & $\begin{array}{l}\text { The portion of porous volume subtracted } \\
\text { from the cell } K \in \mathcal{M} \text { and distributed to } \\
\text { the degree of freedom } \nu \in \Xi_{K} \backslash \mathcal{V}_{\text {ext }} \text {. }\end{array}$ \\
\hline$\phi_{\sigma}$ & $\begin{array}{l}\text { Porous volume associated to the face } \sigma \in \\
\mathcal{F}\end{array}$ \\
\hline$\phi_{\sigma, \mathbf{s}}$ & $\begin{array}{l}\text { The portion of porous volume subtracted } \\
\text { from the fracture face } \sigma \in \mathcal{F}_{\Gamma} \text { and dis- } \\
\text { tributed to the node } \mathrm{s} \in \mathcal{V}_{\sigma} \backslash \mathcal{V}_{\text {ext }} \text {. }\end{array}$ \\
\hline$j_{K}, j_{\sigma}$ & $\begin{array}{l}\text { Rocktype associated with the cell } K \in \mathcal{M} \\
\text { and the fracture face } \sigma \in \mathcal{F}_{\Gamma} \text { respectively. }\end{array}$ \\
\hline$F_{K, \nu}^{\alpha, n}$ & $\begin{array}{l}\text { Discrete flux of the phase } \alpha \text { connecting the } \\
\text { cell } K \text { and the degree of freedom } \nu \in \Xi_{K} \\
\text { at time step } n \text {. }\end{array}$ \\
\hline$F_{\sigma, \mathbf{s}}^{\alpha, n}$ & $\begin{array}{l}\text { Discrete flux of the phase } \alpha \text { connecting the } \\
\text { fracture face } \sigma \text { and the node } \mathbf{s} \in \mathcal{V}_{\sigma} \text { at time } \\
\text { step } n \text {. }\end{array}$ \\
\hline$X_{\mathcal{D}}$ & Vector space of degrees of freedom. \\
\hline$X_{\mathcal{D}}^{0}$ & $\begin{array}{l}\text { The subspace } X_{\mathcal{D}} \text { with homogeneous Dirich- } \\
\text { let boundary conditions. }\end{array}$ \\
\hline \multicolumn{2}{|c|}{ Parametrization of capillary pressure graphs } \\
\hline$\chi_{\nu}$ & $\begin{array}{l}\text { The subset of rocktypes intersecting at the } \\
\text { degree of freedom } \nu \text {. }\end{array}$ \\
\hline $\mathcal{P}_{\chi}$ & $\begin{array}{l}\text { The capillary pressure function associated } \\
\text { with the subset of rocktypes } \chi \text {. }\end{array}$ \\
\hline $\mathcal{S}_{\chi, j}^{g}$ & $\begin{array}{l}\text { The gas saturation function associated with } \\
\text { the subset of rocktypes } \chi \text { and the rocktype } \\
j \in \chi \text {. }\end{array}$ \\
\hline
\end{tabular}

\section{Introduction}

We consider a hybrid dimensional model of two-phase gas-water Darcy flow in fractured porous media. This type of models, introduced in [2], [4] for single phase Darcy flows and in [17], [16], [13], [5] for two-phase Darcy flows, treats fractures as interfaces of co-dimension 1. We will also assume that the pressure of the phases is continuous at the interfaces between the fractures and the matrix domain, which corresponds physically to pervious fractures for which the ratio of the transversal permeability of the fracture to the width of the fracture is large compared with the ratio of the permeability of the matrix to the size of the domain. Note that it does not cover the case of fractures acting as barriers for which the pressure is discontinuous at the matrix fracture interfaces and which are considered in [12], [14], [15], [3], [20] , [18], [1], [6] for single phase flows, and [7] for two phase flows.

In the framework of two-phase Darcy flows in fractured porous media, highly contrasted capillary pressure curves are expected in particular between the matrix and the fractures. Hence, it is crucial to take into account in the model formulation the saturation jumps at the matrix fracture interfaces. In order to do so, as it has been stressed out in [8], the capillary pressure curves have to be extended into the monotone graphs (see e.g. Figures 2).

In several recent works [11], [5], the Vertex Approximate Gradient (VAG) discretization, employing phase pressures formulation, was applied to model two-phase Darcy flows in heterogeneous porous media. In the context of vertex-centered schemes the phase pressures formulation allows to capture the saturation jump condition at the interface between different rocktypes without introducing any additional unknowns at these interfaces. It is, however, limited to strictly increasing capillary pressure curves and lacks robustness compared to pressure-saturation formulations. In this article we extend the scheme introduced in [5] to the case of general increasing capillary pressure curves.

Let $\Omega$ be a bounded domain of $\mathbb{R}^{d}, d=2,3$ assumed to be polyhedral for $d=3$ and polygonal for $d=2$. To fix ideas the dimension will be fixed to $d=3$ when it needs to be specified, for instance in the naming of the geometrical objects or for the space discretization in the next section. The adaptations to the case $d=2$ are straightforward. Let $\bar{\Gamma}=\bigcup_{i \in I} \bar{\Gamma}_{i}$ denotes the network of fractures $\Gamma_{i} \subset \Omega, i \in I$, such that each $\Gamma_{i}$ is a planar polygonal simply connected open domain included in some plane of $\mathbb{R}^{d}$ (see Figure 1).

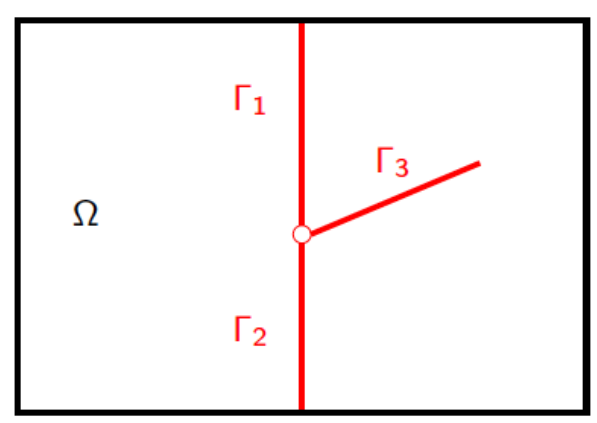

Fig. 1: Example of a 2D domain $\Omega$ with 3 intersecting fractures $\Gamma_{i}, i=1,2,3$.

In the matrix domain $\Omega$ (resp. in the fracture network $\Gamma)$, we denote by $\phi_{m}(\mathbf{x})\left(\operatorname{resp} . \phi_{f}(\mathbf{x})\right)$ the porosity and by $\Lambda_{m}(\mathbf{x})$ (resp. $\left.\Lambda_{f}(\mathbf{x})\right)$ the permeability (resp. tangential permeability) tensor. The thickness of the fractures is denoted by $d_{f}(\mathbf{x})$ for $\mathbf{x} \in \Gamma$. For each phase $\alpha=w, g$ (where $w$ stands for "water" and $g$ for "gas") we denote by $k_{r, m}^{\alpha}(s, \mathbf{x})\left(\operatorname{resp} . k_{r, f}^{\alpha}(s, \mathbf{x})\right)$, the phase relative permeabilities and by $S_{m}^{g}(p, \mathbf{x})\left(\operatorname{resp} . S_{f}^{g}(p, \mathbf{x})\right)$ the possibly set-valued inverses of the monotone graph ex- 
tension of the capillary pressure curves. For $\alpha=w, g$, we will also denote by $\rho^{\alpha}$ the phase densities and by $\mu^{\alpha}$ the phase viscosities which for the sake of clarity are assumed constant.

We denote by $u^{\alpha}$ the pressure of phase $\alpha=w, g$ and by $s_{m}^{g}$ (resp. $s_{f}^{g}$ ) the saturation of gas phase in the matrix (resp. the fracture network) domain. We define the water saturations by

$$
s_{m}^{w}=1-s_{m}^{g} \text { and } s_{f}^{w}=1-s_{f}^{g} .
$$

The Darcy flux of phase $\alpha=w, g$ in the matrix domain is defined by

$$
\mathbf{q}_{m}^{\alpha}=-\frac{k_{r, m}^{\alpha}\left(s_{m}^{\alpha}, \mathbf{x}\right)}{\mu^{\alpha}} \Lambda_{m}\left(\nabla u^{\alpha}-\rho^{\alpha} \mathbf{g}\right),
$$

where $\mathbf{g}=g \nabla z$ stands for the gravity vector. The flow in matrix domain is described by the mass balance equation

$\phi_{m} \partial_{t} s_{m}^{\alpha}+\operatorname{div}\left(\mathbf{q}_{m}^{\alpha}\right)=0$,

and the macroscopic capillary pressure law

$s_{m}^{g} \in S_{m}^{g}\left(u^{g}-u^{w}, \mathbf{x}\right)$.

On each fracture $\Gamma_{i}, i \in I$, we denote formally by $\gamma_{i}$ the trace operator, by $\nabla_{\tau_{i}}$ the tangential gradient and by $\operatorname{div}_{\tau_{i}}$ the tangential divergence. In addition, for all $i \in I$, we can define the two sides \pm of the fracture $\Gamma_{i}$ in $\Omega \backslash \bar{\Gamma}$ and the corresponding unit normal vectors $\mathbf{n}_{i}^{ \pm}$at $\Gamma_{i}$ outward to the sides \pm . Let $\mathbf{q}_{m}^{\alpha, \pm} \cdot \mathbf{n}_{i}^{ \pm}$formally denote the two normal traces of matrix fluxes at both sides of the fracture $\Gamma_{i}$. The Darcy flux of phase $\alpha=w, g$ in the fracture $\Gamma_{i}$ integrated over the width of the fracture is defined by

$$
\mathbf{q}_{f, i}^{\alpha}=-d_{f} \frac{k_{r, f}^{\alpha}\left(s_{f}^{\alpha}, \mathbf{x}\right)}{\mu^{\alpha}} \Lambda_{f}\left(\nabla_{\tau_{i}} \gamma_{i} u^{\alpha}-\rho^{\alpha} \mathbf{g}_{\tau_{i}}\right),
$$

with $\mathbf{g}_{\tau_{i}}=\mathbf{g}-\left(\mathbf{g} \cdot \mathbf{n}_{i}^{+}\right) \mathbf{n}_{i}^{+}$. The flow in each fracture $\Gamma_{i}$ is described by

$d_{f} \phi_{f} \partial_{t} s_{f}^{\alpha}+\operatorname{div}_{\tau_{i}}\left(\mathbf{q}_{f, i}^{\alpha}\right)-\mathbf{q}_{m}^{\alpha,+} \cdot \mathbf{n}_{i}^{+}-\mathbf{q}_{m}^{\alpha,-} \cdot \mathbf{n}_{i}^{-}=0$.

and

$s_{f}^{g} \in S_{f}^{g}\left(\gamma_{i} u^{g}-\gamma_{i} u^{w}, \mathbf{x}\right)$.

The hybrid dimensional two-phase flow model looks for $s_{m}^{g}, s_{f}^{g}$, and $\left(u^{\alpha}\right)_{\alpha=g, w}$ satisfying (1)-(4). In addition to (1)-(4) we prescribe a no-flux boundary conditions at the tips of the immersed fractures, that is to say on $\partial \Gamma \backslash$ $\partial \Omega$, and the mass conservation and pressure continuity conditions at the fracture intersections. We refer to [5] for more details on those conditions. Finally, one should provide some appropriate initial and boundary data.
Remark that for a fixed $\mathbf{x} \in \Omega$ (resp. $\mathbf{x} \in \Gamma$ ) the functions $S_{m}^{g}$ and $S_{f}^{g}$ are, generally speaking, set-valued, this is the case e.g. when the capillary pressure is neglected. Indeed, in such situation the gas saturation takes any value in $[0,1]$ as long as $u^{g}-u^{w}=0$. In addition, $S_{m}^{g}$ and $S_{f}^{g}$ depend on the space variable $\mathbf{x}$ and we will assume that $S_{m}^{g}(\cdot, \mathbf{x})$ is piecewise constant and is defined with respect to a set of so-called rocktypes. Let us denote by $H$ the multi-valued Heavisied function defined by

$$
H(\xi)= \begin{cases}0, & \xi<0 \\ {[0,1],} & \xi=0 \\ 1, & \xi>0\end{cases}
$$

The following assumptions hold on $S_{m}^{g}$ and $S_{f}^{g}$ :

$\left(A_{1}\right) \Omega$ can be decomposed into a set of disjoint connected open polyhedral sets $\left(\Omega_{j}\right)_{j \in J_{m}}$ with $\bigcup_{j \in J_{m}} \overline{\Omega_{j}}=\bar{\Omega}$, such that $S_{m}^{g}(p, \mathbf{x})=S_{m, j}^{g}(p, \mathbf{x})$ for a.e. $\mathbf{x} \in \Omega_{j}$ and all $p \in \mathbb{R}$. Similarly, we suppose that there exists a family of disjoint connected polygonal open sets $\left(\Upsilon_{j}\right)_{j \in J_{f}}$ such that $\bigcup_{j \in J_{f}} \overline{\Upsilon_{j}}=\bar{\Gamma}$ and such that $S_{f}^{g}(p, \mathbf{x})=S_{f, j}^{g}(p)$ for a.e. $\mathbf{x} \in \Upsilon_{j}$ and all $p \in \mathbb{R}$.

$\left(A_{2}\right)$ We assume that for all $l=m, f$ and $j \in J_{l}$ there exist a non decreasing continuous piecewise $C^{1}$ function $a_{l, j}$ from $\mathbb{R}$ to $\mathbb{R}$, a positive integer $r_{l, j}$ and $\left(p_{l, j}^{k}\right)_{k \in\left\{1, \ldots, r_{l, j}\right\}} \in \mathbb{R}^{r_{l, j}},\left(b_{l, j}^{k}\right)_{k \in\left\{1, \ldots, r_{l, j}\right\}} \in\left(\mathbb{R}^{+}\right)^{r_{l, j}}$ such that

$$
S_{l, j}^{g}(p)=a_{l, j}(p)+\sum_{k=1}^{r_{l, j}} b_{l, j, k} H\left(p-p_{l, j, k}\right)
$$

for all $p \in \mathbb{R}$. We also assume that $S_{l, j}^{g}(p) \subset[0,1]$

Remark that the assumption $A_{2}$ allow in particular for negative capillary pressure.

The matrix and fracture relative permeabilities are piecewise constant w.r.t. $\mathbf{x}$ on the same partitions of the matrix and fracture network domains as the capillary pressure curves. In the following, we will denote the mobilities (ratio of the phase relative permeability to the phase viscosity) by $k_{m, j}^{\alpha}(s)$ in the matrix for each rocktype $j \in J_{m}$ and by $k_{f, j}^{\alpha}(s)$ in the fracture network for each rocktype $j \in J_{f}$.

In order to illustrate the difficulty of dealing with both heterogeneous and multi-valued saturation curves $S_{m}^{g}$ and $S_{f}^{g}$, let us admit for the moment that $S_{m}^{g}$ are $S_{f}^{g}$ do not depend on $\mathbf{x}$ and that $S_{m}^{g}(p), S_{f}^{g}(p)$ are singlevalued continuous increasing functions satisfying for $l=$ $m, f$

$$
S_{l}^{g}\left(p \leq p_{\text {ent }, l}\right)=0 \text { and } \lim _{p \rightarrow+\infty} S_{l}^{g}(p)=1
$$


with $p_{e n t, l} \in \mathbb{R}, l=m, f$ been an entry pressure. The figure 2a exhibits a typical form of multi-valued capillary pressure curves corresponding to $S_{m}^{g}(p)$ are $S_{f}^{g}(p)$.

When the system (1) - (4) is solved numerically it is desirable to reduce the number of unknowns by eliminating the algebraic equations (2) and (4), in particular one may expect to have as many as two unknowns by degree of freedom. Note that as long as the functions $S_{l}^{g}, l=m, f$, are single valued (which is the case when the capillary pressure graphs do not have "horizontal" parts) it is possible to express $s_{m}^{g}$ and $s_{f}^{g}$ in terms of $u^{g}$ and $u^{w}$. In other words $\left(u^{w}, u^{g}\right)$ is an admissible couple of primary unknowns. The other admissible couple is $\left(u^{w}, s_{f}^{g}\right)$ since $u^{g}$ and $s_{m}^{g}$ can be expressed as

$$
u^{g}=u^{w}+\left(S_{f}^{g}\right)^{-1}\left(s_{f}^{g}\right) \text { and } s_{m}^{g}=S_{m}^{g} \circ\left(S_{f}^{g}\right)^{-1}\left(s_{f}^{g}\right) .
$$

In contrast, unless $p_{\text {ent }, m}$ is less or equal to $p_{\text {ent, } f \text {, it }}$ is not possible to describe any possible values of $u^{g}$ and $s_{f}^{g}$ at the matrix/fracture interface using the pair $\left(u^{g}, s_{m}^{g}\right)$. However this formulation still can be applied "away" from $\Gamma$.

Let us remark that both $\left(u^{w}, u^{g}\right)$ and $\left(u^{w}, s_{f}^{g}\right)$ formulations lead, after a space-time discretization of (1) and (3), to the equivalent systems of nonlinear algebraic equations. Nevertheless, in practice, the performance of numerical algorithm would heavily depend on the choice of primary variables. In particular it is well known that the use of the formulation based on $u^{g}$ and $u^{w}$ has to be avoided when modeling imbibition in very dry soil. This is explained by the fact that applying the Newton-Raphson method (or some other linearization scheme) for solving nonlinear problems resulting from both formulation breaks the equivalence between nonlinear problems resulting from different formulation.

Next, let's assume that the capillary pressure is neglected in the fracture network domain (see Figure 2b). In that case both $S_{f}^{g}$ and its inverse are set-valued, which in particular implies that neither $\left(u^{w}, s_{f}^{g}\right)$ nor $\left(u^{w}, u^{g}\right)$ can be used as a pair of primary variables for the whole range of values of saturation and capillary pressure. Instead one may switch, as capillary pressure grows, from $\left(u^{w}, s_{f}^{g}\right)$ to $\left(u^{w}, u^{g}\right)$, and even possibly from $\left(u^{w}, u^{g}\right)$ to $\left(u^{w}, s_{m}^{g}\right)$ for $u^{g}-u^{w} \geq p_{\text {ent, } m \text {. Note }}$ that if the capillary pressure in the fracture domain is very small, but not strictly zero one cannot expect the numerical scheme based on $\left(u^{w}, s_{f}^{g}\right)$ formulation to be computationally efficient.

Finally, let us remark that there is no reason to restrict the choice of primary variables to the set of natural variables, that is to say, to $u^{w}, u^{g}, s_{m}^{g}$ and $s_{f}^{g}$. Consider the functions

$\mathcal{P}$ and $\left(\mathcal{S}_{l}^{g}\right)_{l=m, f}$

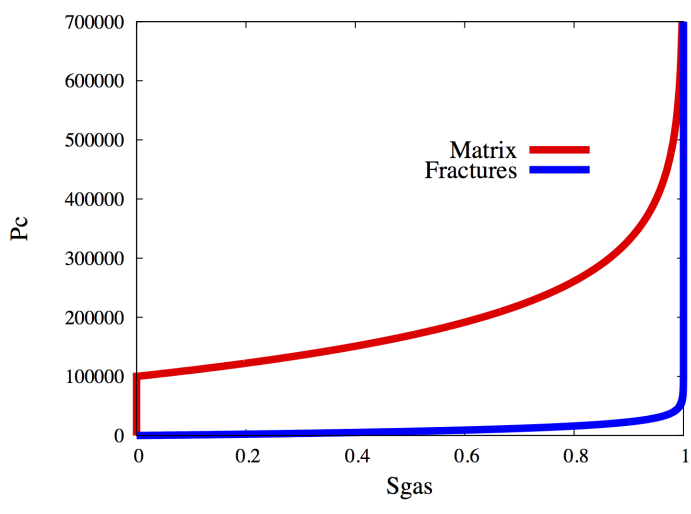

(a) $S_{m}^{g}$ and $S_{f}^{g}$ are single-valued

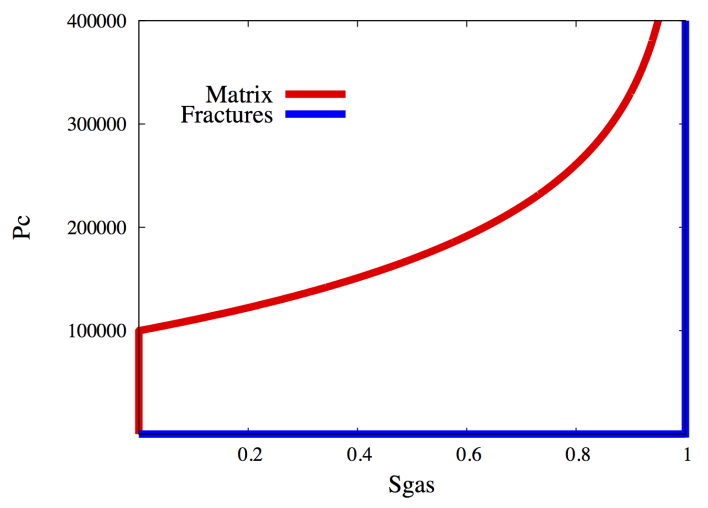

(b) $S_{m}^{g}$ is single-valued, $S_{f}^{g}$ is set-valued

Fig. 2: Typical form of capillary pressure curves in matrix and fracture domains.

defined on an open convex set $\mathcal{I} \subset \mathbb{R}$ such that $\mathcal{P}(\mathcal{I})=$ $\mathbb{R}$ and such that for $\tau \in \mathcal{I}$

$\mathcal{S}_{l}^{g}(\tau) \in S_{l}^{g}(\mathcal{P}(\tau))$ for all $l=m, f$

Then the couple $\left(u^{w}, \tau\right)$ is an admissible couple of primary unknowns with

$$
u^{g}=u^{w}+\mathcal{P}(\tau) \text { and } s_{l}^{g}=\mathcal{S}_{l}^{g}(\tau), l=m, f .
$$

The map $\tau \mapsto\left(\mathcal{P}(\tau), \mathcal{S}_{l}^{g}(\tau)\right), l=m, f$, can be seen as the parametrization of the curve $S_{l}^{g}$. The parametrization (5) is not uniquely defined by (6) even under some additional regularity assumptions (see Proposition 1) and hence one can try to choose the functions $\mathcal{P}(\tau)$ and $\mathcal{S}_{l}^{g}(\tau), l=m, f$, in order to improve the convergence of the nonlinear solver.

The remaining of this article is organized as follows. In the next section we briefly recall the VAG scheme introduced in [5] using $u^{g}$ and $u^{w}$ as primary unknowns. Then we detail the parametrization approach presented above and provide the extension of the VAG discretization accounting for general monotone capillary pressure graphs related to multiple rocktypes, and finally 
we present numerical experiments, which aims to compare the classical pressure-saturation formulations with more advanced parametrizations following the above ideas.

\section{Vertex Approximate Gradient (VAG) Discretization}

In this section we will recall the numerical scheme presented in [5], while assuming that $S_{m}^{g}(\cdot, \mathbf{x})$ and $S_{f}^{g}(\cdot, \mathbf{x})$ are single valued. More precisely, in addition to $\left(A_{2}\right)$ we will assume the following

$\left(A_{2 a}\right)\left(S_{m, j}^{g}\right)_{j \in J_{m}}$ and $\left(S_{f, j}^{g}\right)_{j \in J_{f}}$ are non decreasing continuous piecewise $C^{1}$ functions from $\mathbb{R}$ to $[0,1]$.

The VAG discretization of hybrid dimensional twophase Darcy flows introduced in [5] considers generalised polyhedral meshes of $\Omega$ in the spirit of [10]. Let us briefly recall some notation related to the space discretization. We will denote by $\mathcal{M}$ be the set of disjoint open polyhedral cells, by $\mathcal{F}$ the set of faces and by $\mathcal{V}$ the set of nodes of the mesh. For each cell $K \in \mathcal{M}$ we denote by $\mathcal{F}_{K} \subset \mathcal{F}$ the set of its faces and by $\mathcal{V}_{K}$ the set of its nodes. Similarity, we will denote by $\mathcal{V}_{\sigma}$ the set of nodes of $\sigma \in \mathcal{F}$.

The mesh is supposed to be conforming w.r.t. the fracture network $\Gamma$ in the sense that for all $i \in I$ there exist the subsets $\mathcal{F}_{\Gamma_{i}}$ of $\mathcal{F}$ such that $\bar{\Gamma}_{i}=\bigcup_{\sigma \in \mathcal{F}_{\Gamma_{i}}} \bar{\sigma}$. We will denote by $\mathcal{F}_{\Gamma}$ the set of fracture faces $\bigcup_{i \in I} \mathcal{F}_{\Gamma_{i}}$. The space discretization is also assumed to be compatible with the sets $\left(\Omega_{j}\right)_{j \in J_{m}}$ and $\left(\Upsilon_{j}\right)_{j \in J_{f}}$, that is to say, for all $K \in \mathcal{M}$ there exists $j_{K} \in J_{m}$ such that $K \subset \Omega_{j_{K}}$ and for all $\sigma \in \mathcal{F}_{\Gamma}$ there exists $j_{\sigma} \in J_{f}$ such that $\sigma \subset$ $\Upsilon_{j_{\sigma}}$. In other words, $j_{K}$ is the rocktype of cell $K$ and $j_{\sigma}$ is the rocktype of the fracture face $\sigma$. This geometrical discretization of $\Omega$ and $\Gamma$ is denoted in the following by $\mathcal{D}$. The VAG discretization proposed in [5] is based upon the following vector space of degrees of freedom:

$$
X_{\mathcal{D}}=\left\{v_{K}, v_{\mathbf{s}}, v_{\sigma} \in \mathbb{R}, K \in \mathcal{M}, \mathbf{s} \in \mathcal{V}, \sigma \in \mathcal{F}_{\Gamma}\right\},
$$

and its subspace with homogeneous Dirichlet boundary conditions on $\partial \Omega$ :

$$
X_{\mathcal{D}}^{0}=\left\{v \in X_{\mathcal{D}} \mid v_{\mathbf{s}}=0 \text { for } \mathbf{s} \in \mathcal{V}_{\text {ext }}\right\},
$$

where $\mathcal{V}_{\text {ext }}=\mathcal{V} \cap \partial \Omega$ denotes the set of boundary vertices, and $\mathcal{V}_{\text {int }}=\mathcal{V} \backslash \partial \Omega$ denotes the set of interior vertices. The degrees of freedom are exhibited in Figure 3 for a given cell $K$ with one fracture face $\sigma$ in bold.

The VAG scheme is a control volume scheme in the sense that it results, for each interior degree of freedom and each phase, in a mass balance equation. The two main ingredients are therefore the conservative fluxes and the control volumes. The VAG matrix and fracture fluxes are exhibited in Figure 3. For $u \in X_{\mathcal{D}}$, the matrix fluxes $F_{K, \nu}(u)$ connect the cell $K \in \mathcal{M}$ to the degrees of freedom located at the boundary of $K$, namely $\nu \in$ $\Xi_{K}=\mathcal{V}_{K} \cup\left(\mathcal{F}_{K} \cap \mathcal{F}_{\Gamma}\right)$. The fracture fluxes $F_{\sigma, \mathbf{s}}(u)$ connect each fracture face $\sigma \in \mathcal{F}_{\Gamma}$ to its nodes $\mathbf{s} \in \mathcal{V}_{\sigma}$. Note also that the expression of the matrix (resp. the fracture) fluxes is local to the cell (resp. fracture face) and let us refer to [5] for a more detailed presentation.

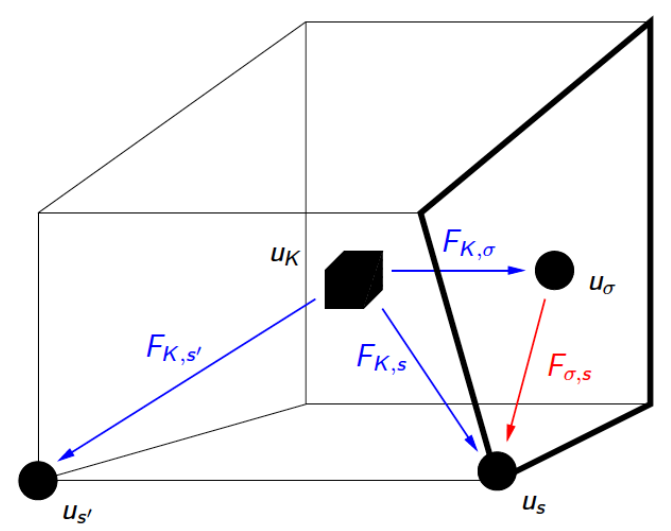

Fig. 3: For a cell $K$ and a fracture face $\sigma$ (in bold), examples of VAG degrees of freedom $u_{K}, u_{\mathbf{s}}, u_{\sigma}, u_{\mathbf{s}^{\prime}}$ and VAG fluxes $F_{K, \sigma}, F_{K, \mathbf{s}}, F_{K, \mathbf{s}^{\prime}}, F_{\sigma, \mathbf{s}}$.

The construction of the control volumes is done by distributing porous volume associated with the cells $K \in \mathcal{M}$ and fracture faces $\sigma \in \mathcal{F}_{\Gamma}$ among the degrees of freedom located on their respective boundaries. For each $K \in \mathcal{M}$ we define a set of non-negative volume fractions $\left(\alpha_{K, \nu}\right)_{\nu \in \Xi_{K} \cap \mathcal{V}_{i n t}}$ satisfying $\sum_{\nu \in \Xi_{K}} \alpha_{K, \nu} \leq 1$, and we set

$$
\begin{gathered}
\phi_{K}=\left(1-\sum_{\nu \in \Xi_{K} \cap \mathcal{V}_{i n t}} \alpha_{K, \nu}\right) \int_{K} \phi_{m}(\mathbf{x}) d \mathbf{x}, \\
\phi_{K, \nu}=\alpha_{K, \nu} \int_{K} \phi_{m}(\mathbf{x}) d \mathbf{x}
\end{gathered}
$$

Similarly, for all $\sigma \in \mathcal{F}_{\Gamma}$ we set

$$
\begin{gathered}
\phi_{\sigma}=\left(1-\sum_{\mathbf{s} \in \mathcal{V}_{\sigma} \cap \mathcal{V}_{i n t}} \alpha_{\sigma, \mathbf{s}}\right) \int_{\sigma} \phi_{f}(\mathbf{x}) d_{f}(\mathbf{x}) d \tau(\mathbf{x}), \\
\phi_{\sigma, s}=\alpha_{\sigma, \mathbf{s}} \int_{\sigma} \phi_{f}(\mathbf{x}) d_{f}(\mathbf{x}) d \tau(\mathbf{x})
\end{gathered}
$$

where we denote by $d \tau(\mathbf{x})$ the $d-1$ dimensional Lebesgue measure on $\Gamma$, and where the volume fractions $\left(\alpha_{\sigma, \mathbf{s}}\right)_{\mathbf{s} \in \mathcal{V}_{\sigma} \cap \mathcal{V}_{i n t}}$ are non-negative and satisfy $\sum_{\mathbf{s} \in \mathcal{V}_{\sigma} \cap \mathcal{V}_{i n t}} \alpha_{\sigma, \mathbf{s}} \leq 1$. 
As it has been shown in [5], the flexibility in the choice of $\alpha_{K, s}$ and $\alpha_{\sigma, s}$ is a crucial asset, compared with usual CVFE approaches and allows to significantly improve the accuracy of the scheme when the permeability field is highly heterogeneous. As exhibited in Figure 4, as opposed to usual CVFE approaches, this flexibility allows to define the control volumes in the fractures with no contribution from the matrix in order to avoid to enlarge artificially the flow path in the fractures.

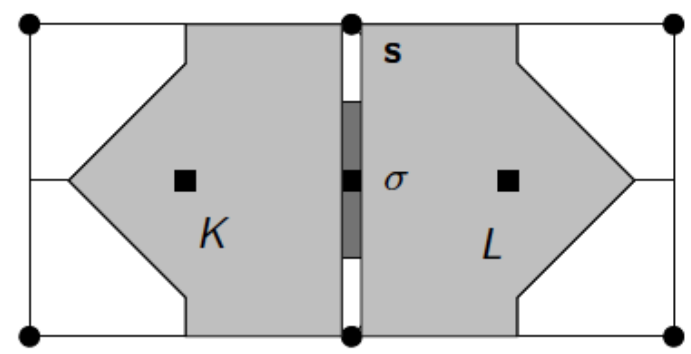

Fig. 4: Example of control volumes at cells, fracture face, and nodes, in the case of two cells $K$ and $L$ splitted by one fracture face $\sigma$ (the width of the fracture has been enlarged in this figure).

For $N \in \mathbb{N}^{*}$, let us consider the time discretization $t^{0}=0<t^{1}<\cdots<t^{n-1}<t^{n} \cdots<t^{N}=T$ of the time interval $[0, T]$. We denote the time steps by $\Delta t^{n}=$ $t^{n}-t^{n-1}$ for all $n=1, \cdots, N$.

Considering homogeneous Dirichlet boundary conditions for convenience, the VAG discretization introduced in [5] of the hybrid dimensional two phase Darcy flow model (1) - (4) looks for $u^{g, n}, u^{w, n} \in X_{\mathcal{D}}^{0}, n=$ $1, \cdots, N$, such that one has for all $v^{\alpha} \in X_{\mathcal{D}}^{0}$ and for $\alpha=g, w:$

$$
\left\{\begin{array}{c}
\sum_{K \in \mathcal{M}}\left(\frac{\phi_{K}}{\Delta t^{n}}\left(S_{K}^{\alpha, n}-S_{K}^{\alpha, n-1}\right)\right. \\
\left.+\sum_{\nu \in \Xi_{K}} k_{m, j_{K}}^{\alpha}\left(S_{K, \nu, u p}^{\alpha, n}\right) F_{K, \nu}^{\alpha, n}\right) v_{K}^{\alpha} \\
+\sum_{K \in \mathcal{M}} \sum_{\nu \in \Xi_{K} \backslash \mathcal{V}_{e x t}}\left(\frac{\phi_{K, \nu}}{\Delta t^{n}}\left(S_{K, \nu}^{\alpha, n}-S_{K, \nu}^{\alpha, n-1}\right)\right. \\
\left.-k_{m, j_{K}}^{\alpha}\left(S_{K, \nu, u p}^{\alpha, n}\right) F_{K, \nu}^{\alpha, n}\right) v_{\nu}^{\alpha} \\
+\sum_{\sigma \in \mathcal{F}_{\Gamma}}\left(\frac{\phi_{\sigma}}{\Delta t^{n}}\left(S_{\sigma}^{\alpha, n}-S_{\sigma}^{\alpha, n-1}\right)\right. \\
\left.+\sum_{\mathbf{s} \in \mathcal{V}_{\sigma}} k_{f, j_{\sigma}}^{\alpha}\left(S_{\sigma, \mathbf{s}, u p}^{\alpha, n}\right) F_{\sigma, \mathbf{s}}^{\alpha, n}\right) v_{\sigma}^{\alpha} \\
\sum_{\sigma \in \mathcal{F}_{\Gamma} \in \mathcal{V}_{\sigma} \backslash \mathcal{V}_{e x t}}\left(\frac{\phi_{\sigma, s}}{\Delta t^{n}}\left(S_{\sigma, \mathbf{s}}^{\alpha, n}-S_{\sigma, \mathbf{s}}^{\alpha, n-1}\right)\right. \\
\left.-k_{f, j_{\sigma}}^{\alpha}\left(S_{\sigma, \mathbf{s}, u p}^{\alpha, n}\right) F_{\sigma, \mathbf{s}}^{\alpha, n}\right) v_{\mathbf{s}}^{\alpha}=0
\end{array}\right.
$$

In (7), the phase fluxes are defined by

$$
\left\{\begin{array}{l}
F_{K, \nu}^{\alpha, n}(u)=F_{K, \nu}\left(u^{\alpha, n}\right)+\rho^{\alpha} g F_{K, \nu}(Z), \\
F_{\sigma, \mathbf{s}}^{\alpha, n}(u)=F_{\sigma, \mathbf{s}}\left(u^{\alpha, n}\right)+\rho^{\alpha} g F_{\sigma, \mathbf{s}}(Z),
\end{array}\right.
$$

with $Z$ denoting the vector $\left(z_{K}, z_{\mathbf{s}}, z_{\sigma}\right)_{K \in \mathcal{M}, \mathbf{s} \in \mathcal{V}, \sigma \in \mathcal{F}_{\Gamma}}$. The upstream values of the saturations $S_{K, \nu, u p}^{\alpha, n}$ and $S_{\sigma, \mathbf{s}, u p}^{\alpha, n}$ are defined by

$$
\left\{\begin{array}{l}
S_{K, \nu, u p}^{\alpha, n}=S_{K}^{\alpha, n} \text { if } F_{K, \nu}^{\alpha}\left(u^{\alpha, n}\right) \geq 0, \\
S_{K, \nu, u p}^{\alpha, n}=S_{K, \nu}^{\alpha, n} \text { if } F_{K, \nu}^{\alpha}\left(u^{\alpha, n}\right)<0,
\end{array}\right.
$$

and

$$
\left\{\begin{array}{l}
S_{\sigma, n}^{\alpha, u p}=S_{\sigma}^{\alpha, n} \text { if } F_{\sigma, \mathbf{s}}^{\alpha}\left(u^{\alpha, n}\right) \geq 0 \\
S_{\sigma, \mathbf{s}, u p}^{\alpha, n}=S_{\sigma, \mathbf{s}}^{\alpha, n} \text { if } F_{\sigma, \mathbf{s}}^{\alpha}\left(u^{\alpha, n}\right)<0 .
\end{array}\right.
$$

As exhibited in Figure 5, the definition of the saturations at the matrix fracture interfaces takes into account the jump of the saturations induced by the different rocktypes. More precisely, for all $K \in \mathcal{M}$ and $\nu \in \Xi_{K} \backslash \mathcal{V}_{\text {ext }}$ we set

$$
\begin{aligned}
& S_{K}^{g, n}=S_{m, j_{K}}^{g}\left(u_{K}^{g, n}-u_{K}^{w, n}\right), \quad S_{K}^{w, n}=1-S_{K}^{g, n}, \\
& S_{K, \nu}^{g, n}=S_{m, j_{K}}^{g}\left(u_{\nu}^{g, n}-u_{\nu}^{w, n}\right), \quad S_{K, \nu}^{w, n}=1-S_{K, \nu}^{g, n},
\end{aligned}
$$

and for all $\sigma \in \mathcal{F}_{\Gamma}$ and $\mathbf{s} \in \mathcal{V}_{\sigma} \backslash \mathcal{V}_{\text {ext }}$ we set

$$
\begin{array}{ll}
S_{\sigma}^{g, n}=S_{f, j_{\sigma}}^{g}\left(u_{\sigma}^{g, n}-u_{\sigma}^{w, n}\right), & S_{\sigma}^{w, n}=1-S_{\sigma}^{g, n}, \\
S_{\sigma, \mathbf{s}}^{g, n}=S_{f, j_{\sigma}}^{g,}\left(u_{\mathbf{s}}^{g, n}-u_{\mathbf{s}}^{w, n}\right), & S_{\sigma, \mathbf{s}}^{w, n}=1-S_{\sigma, \mathbf{s}}^{g, n} .
\end{array}
$$




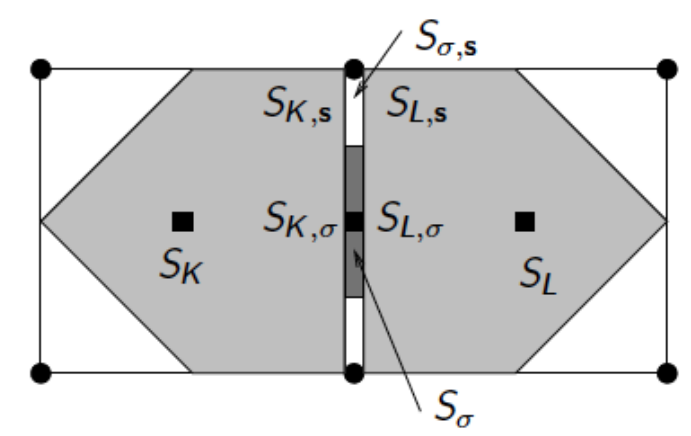

Fig. 5: Saturations inside the cells $K$ and $L$, the fracture face $\sigma$ and at the matrix fracture interfaces taking into account the saturation jumps induced by the different rocktypes.

\section{Parametrization of Saturation Curves at the Rocktype Interfaces}

Now let us demonstrate how the discretization (7)-(11) can be extended to the case of the inverse capillary pressure graphs satisfying $\left(A_{2}\right)$ but not $\left(A_{2 a}\right)$.

Let $J=J_{m} \cup J_{f}$, for all $K \in \mathcal{M}$ we set $\chi_{K}=\left\{j_{K}\right\}$. For all $\sigma \in \mathcal{F}_{\Gamma}$ we set $\chi_{\sigma}=\left\{j_{\sigma}\right\} \cup\left(\bigcup_{\left\{K \mid \sigma \in \mathcal{F}_{K}\right\}}\left\{j_{K}\right\}\right)$. For all $\mathbf{s} \in \mathcal{V}$ we set

$$
\chi_{\mathbf{s}}=\left(\bigcup_{\left\{K \mid \mathbf{s} \in \mathcal{V}_{K}\right\}}\left\{j_{K}\right\}\right) \cup\left(\bigcup_{\left\{\sigma \mid \mathbf{s} \in \mathcal{V}_{\sigma}\right\}}\left\{j_{\sigma}\right\}\right) .
$$

Roughly speaking, for each $\nu \in \mathcal{M} \cup \mathcal{F}_{\Gamma} \cup \mathcal{V}$ the set $\chi_{\nu} \in 2^{J}$ denotes the collection of rocktypes intersecting at the degree of freedom $\nu$. We finally define

$$
\chi_{\mathcal{D}}=\bigcup_{K \in \mathcal{M}}\left\{\chi_{K}\right\} \bigcup_{\sigma \in \mathcal{F}_{\Gamma}}\left\{\chi_{\sigma}\right\} \bigcup_{\mathbf{s} \in \mathcal{V}}\left\{\chi_{\mathbf{s}}\right\},
$$

where $\chi_{\mathcal{D}}$ is seen as a subset of $2^{J}$.

The following proposition justifies the fact that a pair of unknowns is sufficient at any degrees of freedom located on the rocktype intersection.

Proposition 1 Let $\chi \in \chi_{\mathcal{D}}$ and $\left(S_{j}^{g}\right)_{j \in \chi}$ be a finite family of maximal monotone graphs satisfying assumption $A_{2}$ (the second index, $m$ or $f$, is omitted). Then, there exists a family of non decreasing continuous piecewise $C^{1}$ functions $\mathcal{P}_{\chi}$ and $\left(\mathcal{S}_{\chi, j}^{g}\right)_{j \in \chi}$ defined on an open convex set $\mathcal{I} \subset \mathbb{R}$ such that $\mathcal{P}_{\chi}(\mathcal{I})=\mathbb{R}$ and such that for $\tau \in \mathcal{I}$ and $j \in \chi$

$\mathcal{S}_{\chi, j}^{g}(\tau) \in S_{j}^{g}\left(\mathcal{P}_{\chi}(\tau)\right)$

moreover they can be chosen such that the following non-degeneracy condition

$\sum_{j \in \chi} \frac{\mathrm{d}}{\mathrm{d} \tau}\left(\mathcal{S}_{\chi, j}^{g}(\tau), \mathcal{P}_{\chi}(\tau)\right) \neq(0,0)$

is satisfied for a.e. $\tau \in \mathcal{I}$.
Proof : In addition to $A_{2}$ and without loss of generality we may assume that there exist a finite family of real $p_{1}<p_{2}<\ldots<p_{r_{\chi}}$ and a family of non negative $\left(b_{j, k}\right)_{k=1, \ldots, r_{\chi}, j \in \chi}$ such that

$$
S_{j}^{g}(p)=a_{j}(p)+\sum_{k=1}^{r_{\chi}} b_{j, k} H\left(p-p_{k}\right)
$$

for all $p \in \mathbb{R}$ and all $j \in \chi$. Let $\left(\zeta_{k}\right)_{k \in r_{\chi}}$ be a family of non negative real values satisfying $\zeta_{k}=0$ if and only if $\sum_{j \in \chi} b_{j, k}=0$ for all $k=1, \ldots, r_{\chi}$. We set $\mathcal{I}=\mathbb{R}$ and we construct the functions $\mathcal{P}_{\chi}$ and $\left(\mathcal{S}_{\chi, j}^{g}\right)_{j \in \chi}$ as follows. Let $\pi_{1}=p_{1}, \pi_{k}=p_{k}+\sum_{i=1}^{k-1} \zeta_{i}$ for $k=2, \ldots, r_{\chi}$ and $\pi_{r_{\chi}+1}=+\infty$. We set

$\mathcal{P}_{\chi}(\tau)= \begin{cases}\tau, & \tau \leq \pi_{1} \\ p_{k}, & \pi_{k}<\tau \leq \pi_{k}+\zeta_{k}, k=1, \ldots, r_{\chi} \\ p_{k}+\tau- & \pi_{k}-\zeta_{k}, \\ & \pi_{k}+\zeta_{k}<\tau \leq \pi_{k+1}, k=1, \ldots, r_{\chi}\end{cases}$

It is easy to verify that $\mathcal{P}_{\chi}$ is continuous and piecewise $C^{1}$ on $\mathcal{I}$ with $\mathcal{P}_{\chi}(\mathcal{I})=\mathbb{R}$.

For all $k=1, \ldots, r_{\chi}$ and $j \in \chi$ let us denote by $s_{j, k}(p)$ a single valued restriction of $S_{j}^{g}$ on $\left(p_{k}, p_{k+1}\right)$, that is $s_{j, k}(p)=a_{j}(p)+\sum_{i=1}^{k} b_{j, i}$. The restriction of $S_{j}^{g}$ on $\left(-\infty, p_{1}\right)$ will be denoted by $s_{j, 0}(p)=a_{j}(p)$. For any $j \in \chi$ the function $\mathcal{S}_{\chi, j}^{g}$ is defined by

$\mathcal{S}_{\chi, j}^{g}(\tau)=\left\{\begin{array}{c}s_{j, 0}\left(\mathcal{P}_{\chi}(\tau)\right), \quad \tau \leq \pi_{1} \\ s_{j, k-1}\left(p_{k}\right)+\frac{b_{j, k}}{\zeta_{k}}\left(\tau-\pi_{k}\right), \\ \pi_{k}<\tau \leq \pi_{k}+\zeta_{k}, k=1, \ldots, r_{\chi} \\ s_{j, k}\left(\mathcal{P}_{\chi}(\tau)\right), \\ \pi_{k}+\zeta_{k}<\tau \leq \pi_{k+1}, k=1, \ldots, r_{\chi} .\end{array}\right.$

By direct computation one can verify that $\mathcal{S}_{\chi, j}^{g}(\tau)$ is continuous, and that (12) holds. Since each of $s_{j, k}$ is piecewise $C^{1}$ on $\mathcal{I}$ the function $\mathcal{S}_{\chi, j}^{g}(\tau)$ is piecewise $C^{1}$ on $\mathcal{I}$ too. Finally the property (13) is recovered since $s_{j, k}$ and $\mathcal{P}_{\chi}$ are non decreasing and in view of the fact that $\sum_{j \in \chi} b_{j, k}>0$ as soon as the set $\left\{\tau \mid \pi_{k}<\tau \leq\right.$ $\left.\pi_{k}+\zeta_{k}\right\}$ is not empty.

For all $\chi \in \chi_{\mathcal{D}}$ let $\mathcal{P}_{\chi}$ and $\left(\mathcal{S}_{\chi, j}^{g}\right)_{j \in \chi}$ be some family of non decreasing functions associated with the family of graphs $\left(S_{j}^{g}\right)_{j \in \chi}$ and satisfying (12) and (13). The 
saturations are defined by

$$
\left\{\begin{array}{cc}
S_{K}^{g, n}=\mathcal{S}_{\chi_{K}, j_{K}}^{g}\left(\tau_{K}^{n}\right) & \\
S_{K}^{w, n}=1-S_{K}^{g, n} & \text { for all } K \in \mathcal{M}, \\
S_{\sigma}^{g, n}=\mathcal{S}_{\chi_{\sigma}, j_{\sigma}}^{g}\left(\tau_{\sigma}^{n}\right), & \\
S_{\sigma}^{w, n}=1-S_{\sigma}^{g, n} & \text { for all } \sigma \in \mathcal{F}_{\Gamma}, \\
S_{K, \nu}^{g, n}=\mathcal{S}_{\chi_{\nu}, j_{K}}^{g,}\left(\tau_{\nu}^{n}\right), & \\
S_{K, \nu}^{w, n}=1-S_{K, \nu}^{g, n} & \text { for all } K \in \mathcal{M}, \nu \in \Xi_{K}, \\
S_{\sigma, \mathbf{s}}^{g, n}=\mathcal{S}_{\chi_{\mathbf{s}}, j_{\sigma}}^{g}\left(\tau_{\mathbf{s}}^{n}\right), & \\
S_{\sigma, \mathbf{s}}^{w, n}=1-S_{\sigma, \mathbf{s}}^{g, n} & \text { for all } \sigma \in \mathcal{F}_{\Gamma}, \mathbf{s} \in \mathcal{V}_{\sigma},
\end{array}\right.
$$

and the water pressures by

$$
\begin{cases}u_{K}^{w, n}=u_{K}^{g, n}+\mathcal{P}_{\chi_{K}}\left(\tau_{K}^{n}\right) & \text { for all } K \in \mathcal{M} \\ u_{\sigma}^{w, n}=u_{\sigma}^{g, n}+\mathcal{P}_{\chi_{\sigma}}\left(\tau_{\sigma}^{n}\right) & \text { for all } \sigma \in \mathcal{F}_{\Gamma} \\ u_{\mathbf{s}}^{w, n}=u_{\mathbf{s}}^{g, n}+\mathcal{P}_{\chi_{\mathbf{s}}}\left(\tau_{\mathbf{s}}^{n}\right) & \text { for all } \mathbf{s} \in \mathcal{V}\end{cases}
$$

The new scheme consist in finding $u^{g, n}, \tau^{w, n} \in X_{\mathcal{D}}^{0}$, $n=1, \cdots, N$, satisfying (7), (8), (9) along with (14)(15).

\section{Implementation and Numerical Experiments}

In this section we present numerical experiments which aims to compare the robustness and efficiency of the classical pressure-saturation formulation with more advanced choices of primary unknowns which are implemented using the graph parametrization approach presented above. The pressure-pressure formulation is excluded from the comparison since is has a very poor efficiency when dealing with dry $\left(s^{g}\right.$ close to 1$)$ media. In practice, the pressure-pressure formulation has also been tested and it failed to converge for matrix fracture capillarity ratio $\frac{b_{m}}{b_{f}}$ larger than 10 (see below for the definition of this ratio).

We consider only two rocktypes, the matrix rocktype denoted by $j=m$ and the fracture (or fault) rocktype denoted by $j=f$. The phase mobilities are defined for both rocktypes $j=m, f$ and for $\alpha=g, w$ by the following Corey law

$k_{j}^{\alpha}\left(s^{\alpha}, \mathbf{x}\right)=\frac{k_{j, \max }^{\alpha}\left(\bar{s}^{\alpha}\right)^{n_{j}^{\alpha}}}{\mu^{\alpha}}$,

where $\bar{s}^{w}=\frac{s^{w}-s_{r, j}^{w}}{1-s_{r, j}^{w}-s_{r, j}^{g}}$, and $\bar{s}^{g}=\frac{s^{g}-s_{r, j}^{g}}{1-s_{r, j}^{g}-s_{r, j}^{w}}$ are the reduced saturations and $\mu^{\alpha}$ is the phase viscosity.

The capillary pressure/saturation relation are also given for the matrix $(j=m)$ and fracture $(j=f)$ rocktypes by the following Corey law

$S_{j}^{g}(p)= \begin{cases}0 & \text { if } p-p_{\text {ent }, j}<0, \\ 1-e^{-\frac{p-p_{e n t, j}}{b_{j}}} & \text { if } p-p_{\text {ent }, j} \geq 0,\end{cases}$ where the parameter $p_{e n t, j}>0$ stands for the entry pressure. Both $b_{j}$ and $p_{\text {ent }, j}$ depend on the rocktype $j=m, f$. Figure 6 exhibits, the typical shape of the matrix and fracture capillary pressure graphs $P_{c m}$ and $P_{c f}$, which are the multi-valued inverses of $S_{m}^{g}$ and $S_{f}^{g}$ respectively. Remark that, when $b_{j}$ tends to 0 , the graph of $S_{j}^{g}$ tends to the graph of the multi-valued Heaviside function centered at $p=p_{e n t, j}$.
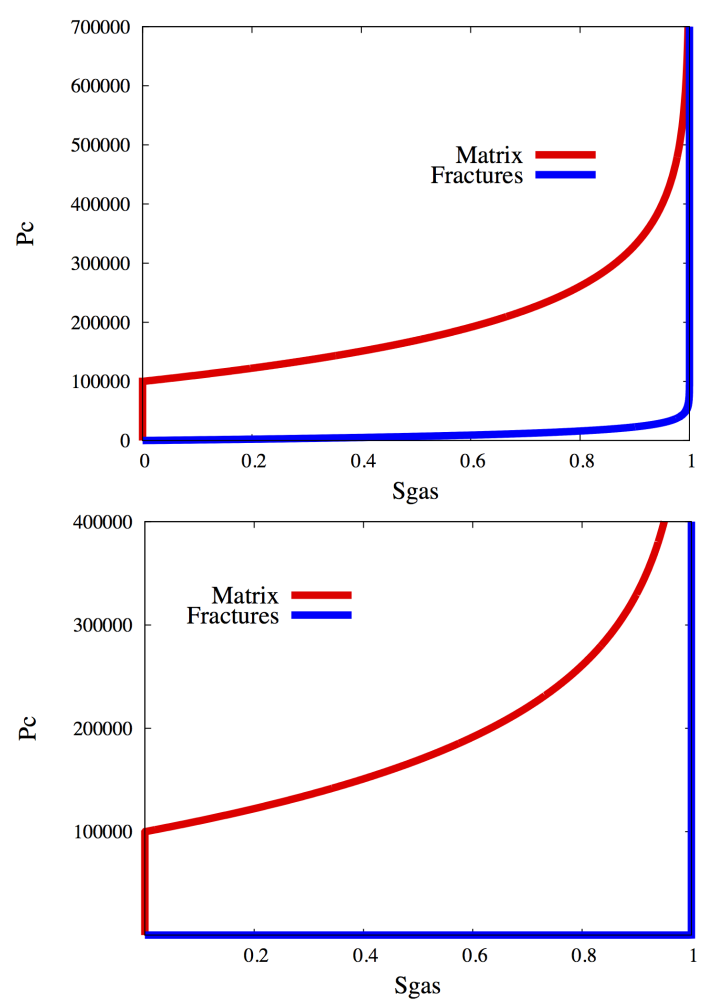

Fig. 6: The graphs of the capillary pressures $P_{c m}$ in the matrix, and $P_{c f}$ in the fractures for $b_{m}=10^{5} \mathrm{~Pa}, b_{f}=10^{4} \mathrm{~Pa}$, $p_{\text {ent }, m}=10^{5} \mathrm{~Pa}, p_{\text {ent }, f}=0$ (left) and $b_{m}=10^{5} \mathrm{~Pa}, b_{f}=0$, $p_{\text {ent }, m}=10^{5} \mathrm{~Pa}, p_{\text {ent }, f}=0$ (right).

Let us remark that the choice of capillary pressure law (17) is mainly motivated by an ease of its implementation and the small number of required parameters. Our method may be extended to other analytical capillary pressure versus saturation relations, such as Van Genuchten law. From the theoretical point of view, the parametrization approach applies as well also to the case of tabulated capillary pressure functions (see Proposition 1). However its practical implementation in that case may be less straightforward, mainly because the choice of the primary unknowns have to be made automatically. The same difficulty arises when more than two rocktypes intersect at the degrees of freedom, which for VAG scheme may happen at the nodal d.o.f. 
The following numerical experiments compare, for different values of the parameters $b_{j}$ and $p_{e n t, j}, j=$ $m, f$, the pressure-saturation formulation using gas pressure and gas saturation as the primary unknowns with some more advanced parametrizations inspired by variable switch techniques. Since only one fracture and one matrix rocktypes are considered, the set $\chi_{\mathcal{D}}$ is equal to

$$
\chi_{\mathcal{D}}=\{\{m\},\{m, f\}\},
$$

where $\chi=\{m\}$ corresponds to degrees of freedom located in the matrix only and $\chi=\{m, f\}$ corresponds to degrees of freedom located at the matrix fracture interfaces. In the following, the primary unknowns for $\chi=\{m\}$ are fixed for both formulations to $\left(u^{g}, s_{m}^{g}\right)$ since this is an efficient and simple choice for a single rocktype. The choices of parametrization at the matrix fracture interfaces i.e. for $\chi=\{m, f\}$ will result in functions $\mathcal{S}_{m}^{g}, \mathcal{S}_{f}^{g}, \mathcal{P}$, which we define below for several types of capillary pressures curves given by Corey law. We will distinguish the following five cases ordered with increasing complexity.

The first two cases, denoted $C_{1}$ and $C_{2}$ assume a zero entry pressure in matrix and fracture network rocktypes and a strictly increasing capillary pressure curve in matrix domain; more precisely we will assume that $b_{m}>0, p_{\text {ent }, m}=p_{\text {ent }, f}=0$ for both cases, and that $0<b_{f}<b_{m}$ for $C_{1}$, while $b_{f}=0$ in the case $C_{2}$.

Then we will address the problem (cases $C_{3}$ and $C_{4}$ ) with a positive entry pressure in the matrix, assuming for both cases that $b_{m}>0, p_{\text {ent }, f}=0$ and that again $0<b_{f}<b_{m}$ for $C_{3}$ and $b_{f}=0$ for $C_{4}$. In addition, as we are interested in large capillary barriers for both $C_{3}$ and $C_{4}$, we will assume that $b_{f}<p_{e n t, m}$.

Finally, the case $C_{5}$ will defined by $p_{\text {ent }, m}>0$, $p_{\text {ent }, f}=0$ and $b_{m}=0=b_{f}=0$.

The choices of the primary unknowns will be compared in terms numerical behavior of the simulations based on the number of linear and nonlinear iterations and on the CPU time.

4.1 Parametrizations for the pressure-saturation and variable-switch formulations

\subsection{1 $C_{1}$ and $C_{2}$ cases}

In this case, the entry pressures are both set to zero i.e. $p_{\text {ent }, j}=0, j=m, f$ leading to the following Corey laws

$S_{j}^{g}(p)= \begin{cases}0 & \text { if } p<0, \\ 1-e^{-\frac{p}{b_{j}}} & \text { if } p \geq 0,\end{cases}$

Figure 7 exhibits the capillary pressure graphs $P_{c m}$ and $P_{c f}$ for $b_{m}=10^{5} \mathrm{~Pa}$ and the ratio $\frac{b_{m}}{b_{f}}=10$.

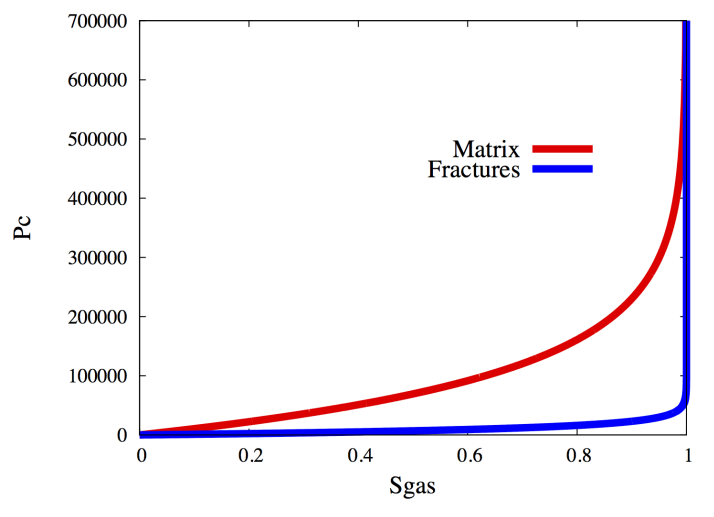

Fig. 7: $P_{c m}$ in the matrix, and $P_{c f}$ in the fractures for $b_{m}=$ $10^{5} \mathrm{~Pa}, b_{f}=10^{4} \mathrm{~Pa}, p_{\text {ent }, m}=p_{\text {ent }, f}=0$.

Pressure-saturation formulation: The formulation is defined by the following set of functions (see the curves on the top of Figure 8)

$$
\left\{\begin{array}{l}
\mathcal{S}_{m}^{g}(\tau)=\tau \\
\mathcal{S}_{f}^{g}(\tau)=P_{c f}^{-1}\left(P_{c m}(\tau)\right)=1-(1-\tau)^{\frac{b_{m}}{b_{f}}} \\
\mathcal{P}(\tau)=P_{c m}(\tau)=-b_{m} \ln (1-\tau)
\end{array}\right.
$$

with $\tau \in[0,1)$.

This formulation can not be applied in the case $C_{2}$, at fact, when $\frac{b_{m}}{b_{f}}$ goes to infinity (i.e. when $b_{m}>0$ while the capillary pressure in the fracture network goes to zero), the function $\mathcal{S}_{f}^{g}(\tau)$ tends to the graph, which is multi-valued at $\tau=0$. Numerically, this would lead to the loss of robustness for large values of $\frac{b_{m}}{b_{f}}$. This pressure-saturation formulation is compared to the variable switch $\left(u^{g}, s_{f}^{g}\right) /\left(u^{g}, s_{m}^{g}\right)$ formulation picking the "steepest" saturation unknown, that is to say the one which has a largest derivative with respect to the capillary pressure. Figure 8 present the curves resulting from both parametrizations for some values of the parameters.

Variable-switch formulation: This formulation is obtained using the relation (12) together with conditions

$$
\max \left(\frac{\mathrm{d} \mathcal{S}_{m}^{g}}{\mathrm{~d} \tau}, \frac{\mathrm{d} \mathcal{S}_{f}^{g}}{\mathrm{~d} \tau}\right)=1
$$

and

$$
\mathcal{S}_{m}^{g}(0)=\mathcal{S}_{f}^{g}(0)=0 .
$$

The computations give

$$
\mathcal{S}_{f}^{g}(\tau)=\left\{\begin{array}{lr}
\tau, & \tau \in\left[0, \tau_{1}\right) \\
P_{c f}^{-1}\left(P _ { c m } \left(\tau-\tau_{1}\right.\right. & \\
\left.\left.\quad+P_{c m}^{-1}\left(P_{c f}\left(\tau_{1}\right)\right)\right)\right) & \\
\quad=1-\left(\tau_{1}+\left(1-\tau_{1}\right)^{\frac{b_{f}}{b_{m}}}-\tau\right)^{\frac{b_{m}}{b_{f}}}, \tau \in\left[\tau_{1}, \tau_{2}\right)
\end{array}\right.
$$


$\mathcal{S}_{m}^{g}(\tau)= \begin{cases}\begin{array}{c}P_{c_{m}}^{-1}\left(P_{c f}(\tau)\right) \\ =1-(1-\tau)^{\frac{b_{f}}{b_{m}}},\end{array} & \tau \in\left[0, \tau_{1}\right), \\ \tau-\tau_{1}+P_{c m}^{-1}\left(P_{c f}\left(\tau_{1}\right)\right) & \\ =\tau-\tau_{1}+1-\left(1-\tau_{1}\right)^{\frac{b_{f}}{b_{m}}}, \tau \in\left[\tau_{1}, \tau_{2}\right),\end{cases}$

and

$\mathcal{P}(\tau)= \begin{cases}P_{c f}\left(\mathcal{S}_{f}^{g}(\tau)\right)=-b_{f} \ln (1-\tau), & \tau \in\left[0, \tau_{1}\right), \\ P_{c m}\left(\mathcal{S}_{m}^{g}(\tau)\right) & \quad=-b_{m} \ln \left(\tau_{1}+\left(1-\tau_{1}\right)^{\frac{b_{f}}{b_{m}}}-\tau\right), \tau \in\left[\tau_{1}, \tau_{2}\right),\end{cases}$

where $\tau_{1}=1-\left(\frac{b_{f}}{b_{m}}\right)^{\frac{b_{m}}{b_{m}-b_{f}}}$ and $\tau_{2}=\tau_{1}+\left(1-\tau_{1}\right)^{\frac{b_{f}}{b_{m}}}$. It is worth noticing that, by construction, the derivatives of the functions $\mathcal{S}_{f}^{g}(\tau), \mathcal{S}_{m}^{g}(\tau)$ and $\mathcal{P}(\tau)$ are continuous at $\tau=\tau_{1}$ and that $\frac{\mathrm{d} \mathcal{S}_{m}^{g}}{\mathrm{~d} \tau}\left(\tau_{1}\right)=\frac{\mathrm{d} \mathcal{S}_{f}^{g}}{\mathrm{~d} \tau}\left(\tau_{1}\right)=1$. When, for a fixed $b_{m}$, the ratio $\frac{b_{m}}{b_{f}}$ goes to infinity, the variable switch parametrization tends to the following formulation (see Figure 9):

$\mathcal{S}_{f}^{g}(\tau)=\left\{\begin{array}{l}\tau, \tau \in\left[0, \tau_{1}\right), \\ 1, \tau \in\left[\tau_{1}, \tau_{2}\right),\end{array}\right.$

$\mathcal{S}_{m}^{g}(\tau)= \begin{cases}0, & \tau \in\left[0, \tau_{1}\right), \\ \tau-\tau_{1}, & \tau \in\left[\tau_{1}, \tau_{2}\right),\end{cases}$

and

$\mathcal{P}(\tau)= \begin{cases}0, & \tau \in\left[0, \tau_{1}\right), \\ P_{c m}\left(\mathcal{S}_{m}^{g}(\tau)\right. & \\ =-b_{m} \ln \left(1-\left(\tau-\tau_{1}\right)\right), & \tau \in\left[\tau_{1}, \tau_{2}\right),\end{cases}$

with $\tau_{1}=1, \tau_{2}=2$. Note that this limit case of a vanishing capillary pressure in the fractures cannot be accounted for by the pressure-saturation formulation.

\subsection{2 $C_{3}$ and $C_{4}$ cases}

Next we consider the test cases with non zero entry pressure in the matrix $p_{\text {ent }, m}>0$ and with zero entry pressure in the fractures $p_{e n t, f}=0$. The graphs of $P_{c j}$, $j=m, f$ are represented in Figure 6 for $p_{\text {ent }, m}=10^{5}$ $\mathrm{Pa}, b_{m}=10^{5} \mathrm{~Pa}$, and $b_{f}=10^{4} \mathrm{~Pa}$.

Pressure-saturation formulation: At the matrix fracture interfaces the capillary pressure (see Figure 6) can not be expressed as a function of $s_{m}^{g}$ for $p<p_{\text {ent }, m}$, but it is however a function of $s_{f}^{g}$ as long as $b_{f}>0$. Hence we choose $s_{f}^{g}$ as primary unknown at the matrix fracture interfaces, which leads to

$$
\mathcal{S}_{f}^{g}(\tau)=\tau, \quad \mathcal{P}_{m}(\tau)=\left(S_{f}^{g}\right)^{-1}(\tau)=-b_{f} \ln (1-\tau),
$$

and

$$
\mathcal{S}_{m}^{g}(\tau)=\left\{\begin{array}{c}
0, \quad \tau<S_{f}^{g}\left(p_{\text {ent }, m}\right)=1-e^{\frac{-p_{e n t, m}}{b_{f}}}, \\
P_{c m}^{-1}\left(P_{c f}(\tau)\right) \\
=1-e^{\frac{p_{e n t, m}}{b_{m}}}(1-\tau)^{\frac{b_{f}}{b_{m}}}, \\
\tau \geq 1-e^{\frac{-p_{e n t, m}}{b_{f}}} .
\end{array}\right.
$$

As in the previous case the pressure-saturation formulation can not be extended to the degenerate case $C_{4}$. Remark that when $\frac{b_{m}}{b_{f}}$ goes to infinity the function $P_{c m}^{-1}\left(P_{c f}(\tau)\right)$ becomes multi-valued at $\tau=1$ (see Figure 10), which results in severe numerical instabilities. As a result, we were unable to obtain the convergence of the nonlinear solver for $\frac{b_{m}}{b_{f}}>10$.

Variable-switch formulation: When $b_{f}=0$ it is clear that the capillary pressure is no longer a function of the saturation for its values in the interval $\left(0, p_{\text {ent }, m}\right)$. At fact, for those values of capillary pressure the relevant pair of unknowns is $\left(u^{w}, u^{g}\right)$ or $\left(u^{w}, u^{g}-u^{w}\right)$. As we are interested in treating the limit case $b_{f}=0$ we investigate the following formulation (see Figure 10), which roughly speaking switches between the primary variables $\left(u^{g}, s_{f}^{g}\right),\left(u^{g}, u^{g}-u^{w}\right)$, and $\left(u^{g}, s_{m}^{g}\right)$

$\mathcal{S}_{f}^{g}(\tau)=\left\{\begin{array}{cr}\tau, & \tau \in\left[0, \tau_{1}\right), \\ P_{c f}^{-1}\left(p_{\text {ent }, m}\left(\tau-\tau_{1}\right)+P_{c f}\left(\tau_{1}\right)\right) & \\ =1-\left(1-\tau_{1}\right) e^{-\frac{p_{e n t, m}}{b_{f}}\left(\tau-\tau_{1}\right)}, & \tau \in\left[\tau_{1}, \tau_{2}\right), \\ P_{c f}^{-1}\left(P_{c m}\left(\tau-\tau_{2}\right)\right) & \\ =1-\left(1-\left(\tau-\tau_{2}\right)\right)^{\frac{b_{m}}{b_{f}}} e^{\frac{-p_{e n t, m}}{b_{f}}} & \tau \in\left[\tau_{2}, \tau_{3}\right),\end{array}\right.$

$\mathcal{S}_{m}^{g}(\tau)= \begin{cases}0, & \tau \in\left[0, \tau_{2}\right), \\ \tau-\tau_{2}, & \tau \in\left[\tau_{2}, \tau_{3}\right],\end{cases}$

and

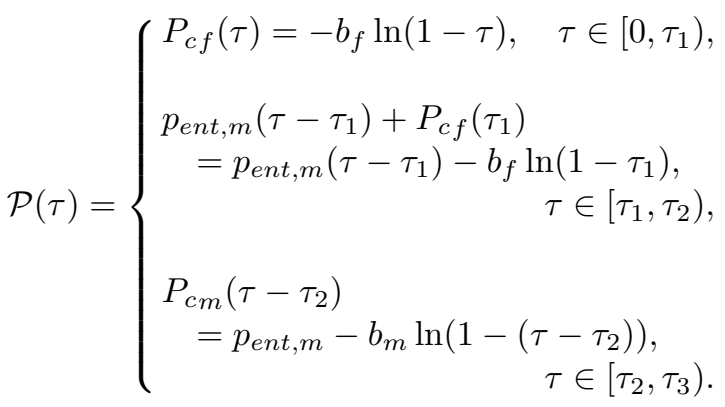

The value $\tau_{1}=1-\frac{b_{f}}{p_{e n t, m}}$ used for the switch between $\left(u^{g}, s_{f}^{g}\right)$ and $\left(u^{g}, u^{g}-u^{w}\right)$, and the value $\tau_{2}=\tau_{1}+$ $1-\frac{P_{c f}\left(\tau_{1}\right)}{p_{e n t, m}}$ used for the switch between $\left(u^{g}, u^{g}-u^{w}\right)$ and 

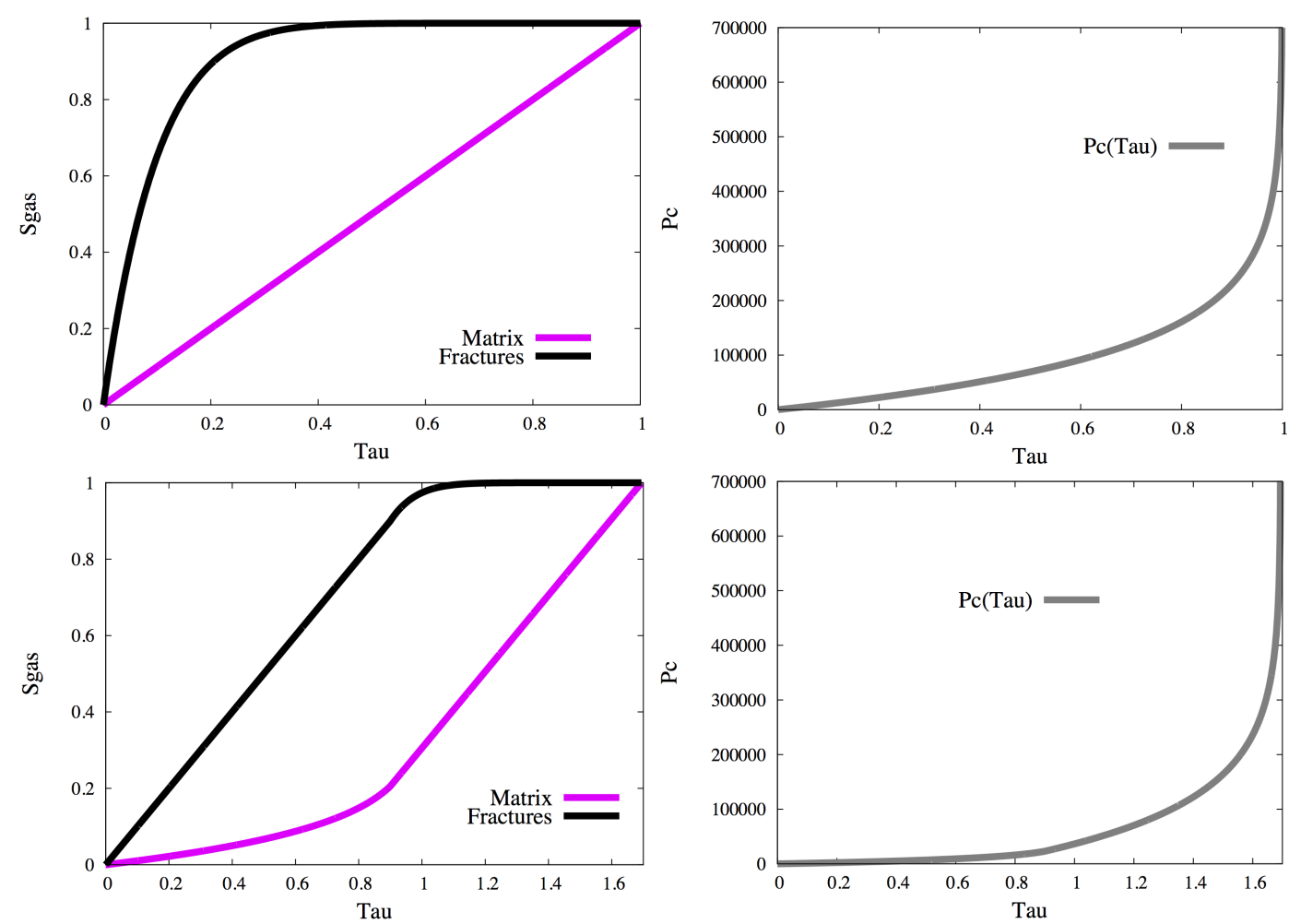

Fig. 8: $\mathcal{S}_{m}^{g}, \mathcal{S}_{f}^{g}$, and $\mathcal{P}$ curves for the pressure-saturation (on the top) and variable switch formulation (at the bottom) for $b_{m}=10^{5} \mathrm{~Pa}, b_{f}=10^{4} \mathrm{~Pa}$, and $p_{\text {ent }, m}=p_{\text {ent }, f}=0$.
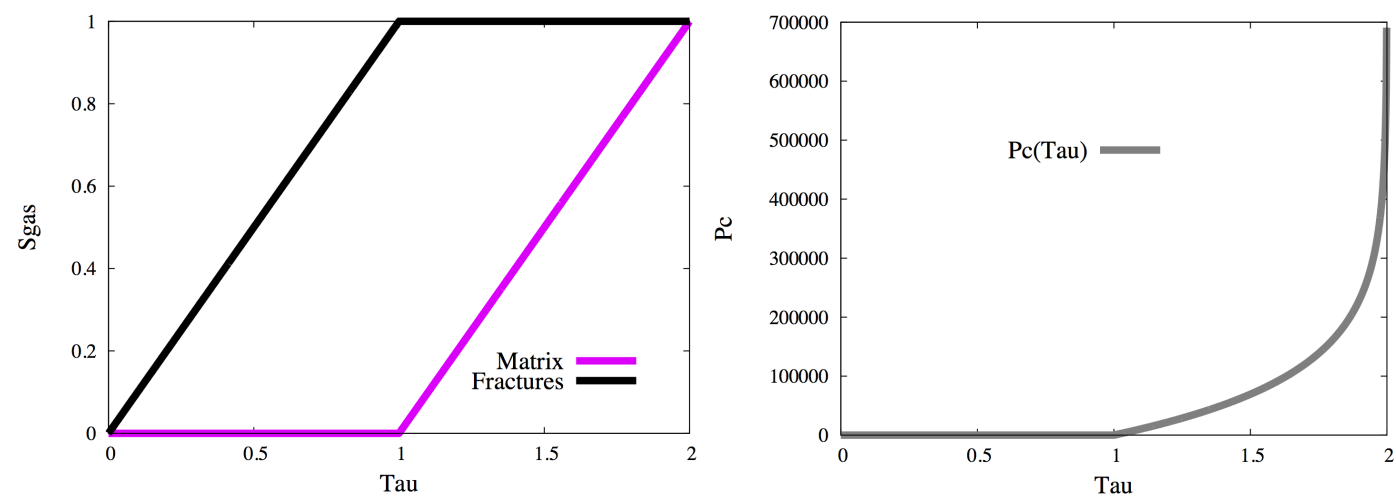

Fig. 9: $\mathcal{S}_{m}^{g}, \mathcal{S}_{f}^{g}$, and $\mathcal{P}$ curves for the variable switch formulation and $b_{m}=10^{5} \mathrm{~Pa}, b_{f}=0$, and $p_{\text {ent }, m}=p_{\text {ent }, f}=0$.

$\left(u^{g}, s_{m}^{g}\right)$ are chosen such that the derivatives of the functions $\mathcal{S}_{f}^{g}(\tau), \mathcal{S}_{m}^{g}(\tau), \mathcal{P}(\tau)$ remain continuous on $\left[0, \tau_{3}\right)$, with $\tau_{3}=\tau_{2}+1$. Remark that $P_{c f}\left(\tau_{1}\right)<p_{\text {ent }, m}$ thanks to the assumption $b_{f}<p_{\text {ent }, m}$ and that $\mathcal{P}\left(\tau_{2}\right)=p_{\text {ent }, m}$. When the ratio $\frac{b_{m}}{b_{f}}$ goes to infinity the following formulas are recovered

$\mathcal{S}_{f}^{g}(\tau)=\left\{\begin{array}{l}\tau, \tau \in\left[0, \tau_{1}\right), \\ 1, \tau \in\left[\tau_{1}, \tau_{3}\right)\end{array}\right.$

$\mathcal{S}_{m}^{g}(\tau)= \begin{cases}0, & \tau \in\left[0, \tau_{2}\right), \\ \tau-\tau_{2}, & \tau \in\left[\tau_{2}, \tau_{3}\right),\end{cases}$ and

$\mathcal{P}(\tau)=\left\{\begin{array}{lr}0, & \tau \in\left[0, \tau_{1}\right), \\ p_{\text {ent }, m}\left(\tau-\tau_{1}\right), & \tau \in\left[\tau_{1}, \tau_{2}\right), \\ P_{c m}\left(\mathcal{S}_{m}^{g}(\tau)\right. & \\ =-b_{m} \ln \left(1-\left(\tau-\tau_{2}\right)\right)+p_{\text {ent }, m}, \\ \tau \in\left[\tau_{2}, \tau_{3}\right),\end{array}\right.$

where $\tau_{1}=1, \tau_{2}=2$, and $\tau_{3}=3$. Remark that for $b_{f}=$ 0 and $\tau \in\left[\tau_{1}, \tau_{2}\right]$ the matrix rocktype acts as a barrier for both phases so that none of them can penetrate it unless the capillary pressure rises or drops enough. 

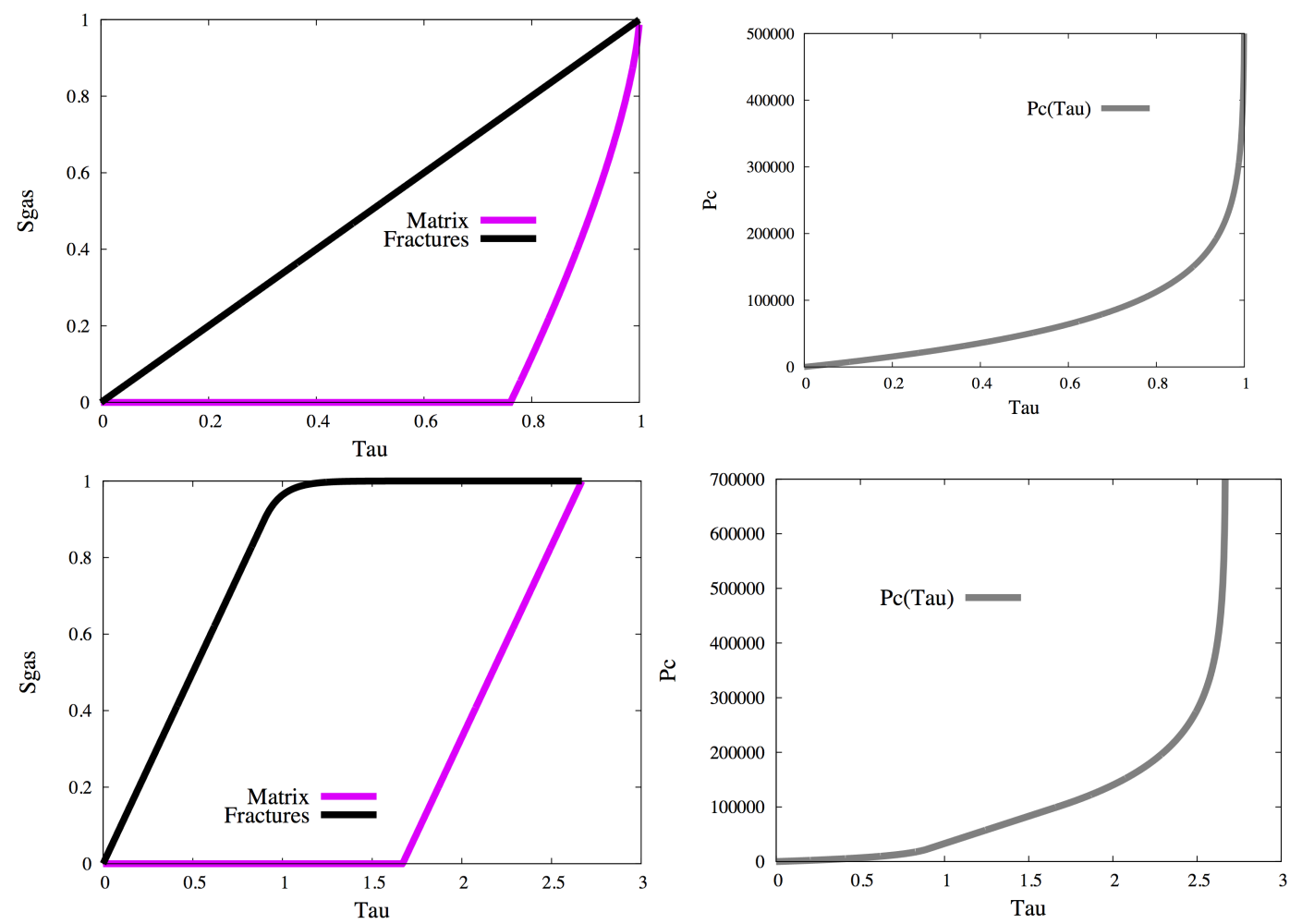

Fig. 10: $\mathcal{S}_{m}^{g}, \mathcal{S}_{f}^{g}$, and $\mathcal{P}$ curves for the pressure-saturation formulation (on the top) and variable switch formulation (at the bottom) for $b_{m}=10^{5} \mathrm{~Pa}, b_{f}=10^{4} \mathrm{~Pa}, p_{\text {ent }, m}=10^{5} \mathrm{~Pa}, p_{\text {ent }, f}=0$.

\subsection{3 $C_{5}$ case}

Finally we consider the case $b_{m}=b_{f}=0$, and $p_{\text {ent }, m}>$ 0 , and $p_{\text {ent }, f}=0$ (see Figure 11). This test case can only be treated using the formulation involving multiple primary variable switches.

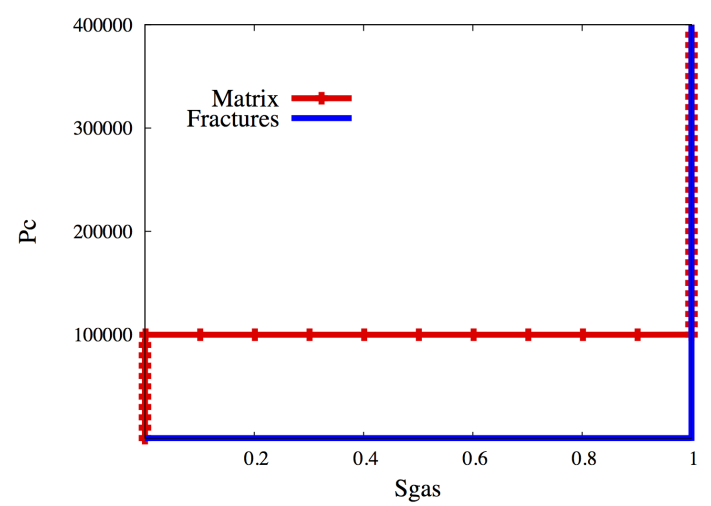

Fig. 11: The capillary pressure curves $P_{c m}$ in the matrix and $P_{c f}$ in the fractures for $b_{m}=b_{f}=0, p_{e n t, m}=10^{5} \mathrm{~Pa}$, and $p_{\text {ent }, f}=0$.
In the spirit of the previous case we define the following parametrization:

$\mathcal{S}_{f}^{g}(\tau)=\left\{\begin{array}{l}\tau, \tau \in\left[0, \tau_{1}\right), \\ 1, \tau \in\left[\tau_{1}, \tau_{3}\right)\end{array}\right.$

$\mathcal{S}_{m}^{g}(\tau)= \begin{cases}0, & \tau \in\left[0, \tau_{2}\right), \\ \tau-\tau_{2}, & \tau \in\left[\tau_{2}, \tau_{3}\right],\end{cases}$

and

$\mathcal{P}(\tau)= \begin{cases}0, & \tau \in\left[0, \tau_{1}\right), \\ p_{\text {ent }, m}\left(\tau-\tau_{1}\right), & \tau \in\left[\tau_{1}, \tau_{2}\right), \\ p_{\text {ent }, m}, & \tau \in\left[\tau_{2}, \tau_{3}\right],\end{cases}$

with $\tau_{1}=1, \tau_{2}=2$, and $\tau_{3}=3$.

\subsection{Tight gas test case}

The family of test cases presented here simulates the liquid gas two phase Darcy flow in a tight gas reservoir. The data set is similar to Example 2 of [9] except for the choice of the capillary pressure curves. The reservoir is defined by the domain $\Omega=(-500,500) \times$ $(-250,250) \times(-100,100)$ (in meters). Three transverse 


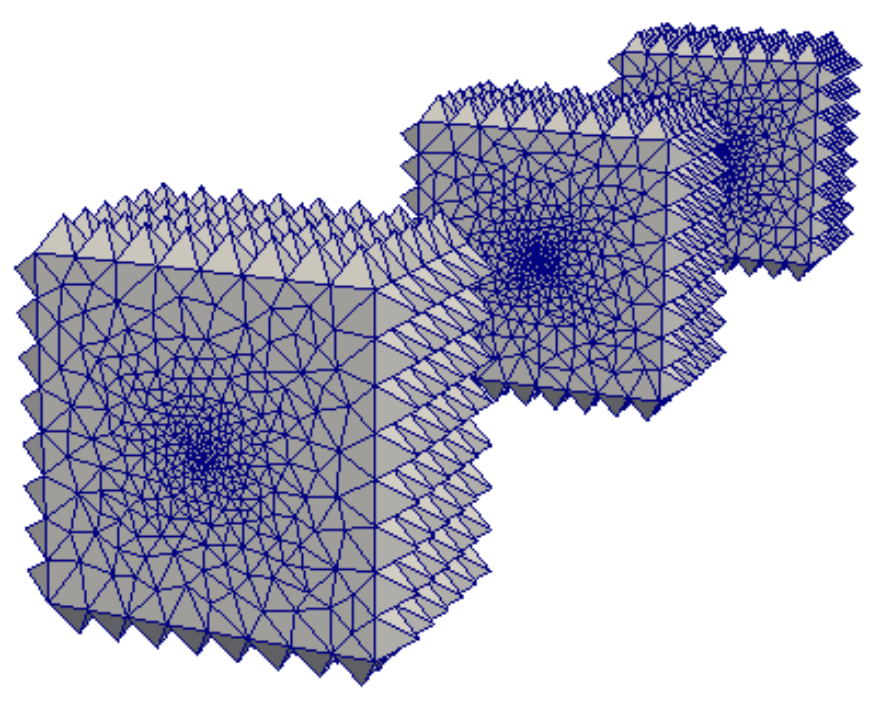

Fig. 12: The prismatic meshes with their layer of pyramids for each refined box around each fracture located at the center of each box (the thickness of the prisms and pyramids has been enlarged for the sake of clarity). fractures $\Gamma_{i}, i=1,2,3$ of width $d_{f}=0.02 \mathrm{~m}$ are initiated by hydraulic fracturing from a horizontal well. They are defined by the squares $\left\{x_{i}\right\} \times(-50,50) \times$ $(-50 \times 50)$ with $x_{1}=-250, x_{2}=0, x_{3}=+250$. An horizontal well of radius $r_{w}=0.1 \mathrm{~m}$ is located along $y=z=0$ and perforates each fracture $\Gamma_{i}, i=1,2,3$ in a triangular equilateral face of center $x_{i}, y=z=0$ and of edge size $1 \mathrm{~m}$. During the water injection phase, the water penetrates only a few tens of centimeters in the matrix due to the low permeability of the reservoir. Therefore in order to obtain an accurate water saturation in the neighbourhood of the fractures with a reasonable number of cells, a strong anisotropic refinement is needed in the normal direction in the neihbourhood of each fracture. As exhibited in Figures 12 and 13 this anisotropic refinement is obtained using prismatic meshes with triangular base. In order to match the boundaries of these refined boxes with the surrounding tetrahedral mesh of the reservoir, a layer of pyramids is added around each fracture box as exhibited in Figure 12. The tetrahedral mesh matching the triangulation of the fracture box boundaries has been obtained using TetGen [19]. Table 1 summarizes the characteristics of the resulting hybrid mesh that will be used in the following numerical test cases. This mesh includes ten layers of prisms of thickness $0.1 \mathrm{~m}$ on both sides of each fracture.

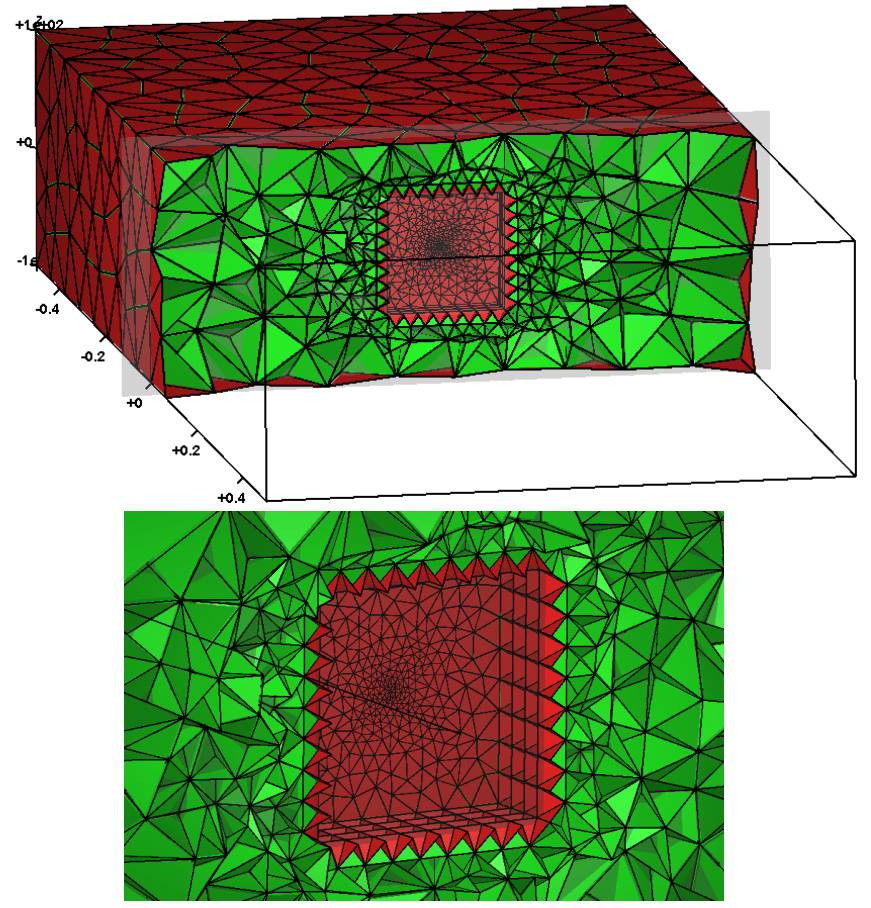

Fig. 13: Connection of the prismatic mesh around one fracture with the surrounding tetrahedral mesh using a layer of pyramids (the thickness of the prisms and pyramids has been enlarged for the sake of clarity).

\begin{tabular}{|c|c|c|c|}
\hline $\mathbf{N}_{\text {cells }}$ & $\mathbf{N}_{\text {nodes }}$ & $\mathbf{N}_{\text {FracF }}$ & Linear system d.o.f. \\
\hline 232920 & 45193 & 1634 & 46827 \\
\hline
\end{tabular}

Table 1: Number $N_{\text {cells }}$ of cells, number $N_{\text {nodes }}$ of nodes, number $N_{\text {FracF }}$ of fracture faces and number of d.o.f. in the linear system after elimination of the cell d.o.f. (2 physical unknowns per d.o.f.).

In this test case the mobilities are defined by $s_{r, m}^{w}=$ $0.2, s_{r, m}^{g}=0, k_{m, \max }^{w}=0.3, k_{m, \max }^{g}=0.6, n_{m}^{w}=$ $1.5, n_{m}^{g}=3$ in the matrix, and by $s_{r, f}^{w}=s_{r, f}^{g}=0$, $k_{f, \text { max }}^{w}=k_{f, \text { max }}^{g}=1, n_{f}^{w}=n_{f}^{g}=1$ in the fractures. The parameters of the Corey capillary pressures are fixed to $b_{m}=10^{5} \mathrm{~Pa}$ for cases $C_{1}, C_{2}, C_{3}$ and $C_{4}$, and to $p_{\text {ent, } m}=10^{5} \mathrm{~Pa}$ for cases $C_{3}, C_{4}$, and $C_{5}$. For cases $C_{1}$ and $C_{3}$ we will investigate the set of values $b_{f}=1,10,10^{2}, 10^{3}, 10^{4} \mathrm{~Pa}$ in the fractures.

The viscosities of the two phases are set to $\mu^{w}=$ $10^{-3} \mu^{g}=2.3510^{-5} \mathrm{~Pa} \cdot \mathrm{s}$, and their densities are fixed to the constant value $\rho^{w}=1000 \mathrm{~kg} / \mathrm{m}^{3}$ for the water phase, and to the perfect gas density $\rho^{g}\left(u^{g}\right)=\frac{M}{R T} u^{g}$ $\mathrm{kg} / \mathrm{m}^{3}$ for the gas phase with $M=0.016 \mathrm{Kg}$ corresponding to methane and $R=8.32 \mathrm{~J} \cdot \mathrm{mol}^{-1} \cdot \mathrm{K}^{-1}$. The reservoir is initially at the liquid pressure $u^{w}=40010^{5}$ $\mathrm{Pa}$, at the residual water saturation in the matrix and at water saturation close to 0 in the fractures obtained by 
the continuity of the capillary pressure at the matrix fracture interfaces. The permeability of the matrix is isotropic and given by $\Lambda_{m}=\lambda_{m}$ Id with $\lambda_{m}=210^{-17}$ $\mathrm{m}^{2}$, very low compared with the permeability of the fractures $\Lambda_{f}=\lambda_{f}$ Id with $\lambda_{f}=210^{-12} \mathrm{~m}^{2}$. The porosity is equal to $\phi_{m}=0.1$ in the matrix and to $\phi_{f}=0.3$ in the fractures.

The liquid is first injected at high hydraulic fracturing pressure $100010^{5} \mathrm{~Pa}$ fixed at each perforation during 1 day. This high pressure water injection phase mimics indeed the invasion of the matrix by fracturing fluid during hydraulic fracturing operations. After injection, the well is closed during the next three days. The well is then put in production at a constant bottom pressure of $30010^{5} \mathrm{~Pa}$ during the next 296 days. Only a part of the invaded water will be back-produced in this early phase of production. The simulation runs over a period of 300 days and the nonlinear systems obtained at each time step are solved by a NewtonRaphson method. The time stepping is defined by an initial time step of 0.001 hour and a maximum time step of 0.05 days during the water injection period, of 0.1 days during the well closure, and of 5 days during the production period. If the Newton method does not converge after 35 iterations, the time step is chopped by a factor 2 and recomputed. The time step is increased by a factor 1.2 after each successful time step until it reaches the maximum time step. The stopping criteria on the relative residuals are fixed to $10^{-6}$ for the GMRes solver preconditioned by ILU0 and to $10^{-5}$ for the Newton method.

Finally, let us remark that the variable switch formulations introduced in Section 4.1 result in Lipschitz continuous saturation functions $\mathcal{S}_{m}^{g}$ and $\mathcal{S}_{f}^{g}$ (with Lipschitz constant less or equal to 1 ). This is an advantage compare to classical pressure-saturation formulation. However, the second derivative of the functions $\mathcal{S}_{m}^{g}$ and $\mathcal{S}_{f}^{g}$ may become arbitrarily large depending on the values of the capillary curve parameters $b_{m}$ and $b_{f}$. Indeed, the lack of smoothness occurs at the switching values of the parameter $\tau$, namely at $\tau_{1}$ for the cases $C_{1}$ and $C_{2}$, and at $\tau_{1}, \tau_{2}$ for the cases $C_{3}-C_{5}$. In order to deal with this issue the Newton iterations are damped using the following rule: if the Newton iterate for $\tau$ variable tries to jump from below to above the value $\tau_{i}=\tau_{1}, \tau_{2}$ (or from above to below), it is projected onto $\tau=\tau_{i}-\frac{\epsilon}{2}\left(\tau=\tau_{i}+\frac{\epsilon}{2}\right.$ correspondingly). More precisely, let $\epsilon>0$, and let $\tau_{\nu}^{k}, \Delta \tau_{\nu}^{k}$ denote the value of the unknown and its increment at the degree of freedom $\nu$, at the iteration $k$ of nonlinear solver. If

$$
\tau_{\nu}^{k}<\tau_{i}-\epsilon \text { and } \tau_{\nu}^{k}+\Delta \tau_{\nu}^{k}>\tau_{i}
$$

we set $\tau_{\nu}^{k+1}=\tau_{i}-\frac{\epsilon}{2}$, otherwise we set $\tau_{\nu}^{k+1}=\tau_{\nu}^{k}+$ $\Delta \tau_{\nu}^{k}$. Similarly the Newton iteration is damped as soon as $\tau_{\nu}^{k}>\tau_{i}+\epsilon$ and $\tau_{\nu}^{k}+\Delta \tau_{\nu}^{k}<\tau_{i}$. Compare to the straightforward Newton's method, this procedure may result in a slightly larger number of iterations. In return it increases the robustness of the nonlinear solver. In the following numerical experiments we have taken $\epsilon=$ $10^{-5}$.

The numerical behaviour of the simulations for both variable switch and pressure-saturation formulations (cases $C_{1}$ and $C_{3}$ ), and for the variable switch formulation only (cases $C_{2}, C_{4}$ and $C_{5}$ ), is exhibited in Tables 2, 3 and 4. These tables present the number of successful time steps, the number of time step chops, the number of Newton iterations by successful time steps, the number of GMRes iterations by Newton iteration, and the CPU time. Figure 14 exhibits in case $C_{1}$ for $\frac{b_{m}}{b_{f}}=1000$ the cumulated number of Newton iterations as a function of time for the pressure-saturation and variable switch formulations. It is clear that most of the time step failures occur for the pressure-saturation formulation during the water injection period. Figure 14 also plots, for the same test case, CFL numbers in the matrix and in the fractures as a function of time obtained with the variable switch formulation. These CFL numbers correspond to the one obtained by an explicit Euler integration of the scalar hyperbolic equation for the saturation unknown using the total velocity and the fractional flow. It illustrates that the time steps used in this simulation are many orders of magnitude larger than the ones obtained using an IMPES scheme both in the fractures and in the matrix.

The variable switch formulation turns out to be more efficient and more robust w.r.t. the value of $\frac{b_{m}}{b_{f}}$ both in terms of number of Newton iterations and the number of time step chops. Note that, as it can be expected, for $\frac{b_{m}}{b_{f}}=\infty$ the variable switch formulation performs similarly as for the case $\frac{b_{m}}{b_{f}}=10^{5}$. As shown in Table 3 , in the case of the entry pressure $p_{\text {ent }, m}=10^{5}$ $\mathrm{Pa}$, the pressure-saturation formulation fails to converge except for small ratio $\frac{b_{m}}{b_{f}}$. Table 4 shows that the variable switch formulation still performs efficiently in the degenerate case $C_{5}$.

Figures 15 and 16 exhibit, at the end of each simulation period at $t=1$ day, $t=4$ days and $t=300$ days, the water saturation in the perforated face in the fracture and the cut of the water saturation in the matrix along the line $y=z=0$ as a function of the distance to the fracture. One clearly sees that the water phase fills the fractures during the water injection period and penetrates the matrix less than one meter from the fractures. At the end of the well closure period, water has been sucked by imbibition from the fractures to the 


\begin{tabular}{|c|c|c|c|c|c|c|c|c|c|c|}
\hline & \multicolumn{5}{|c|}{ pressure-saturation } & \multicolumn{5}{|c|}{ variable switch } \\
\hline$\frac{b_{m}}{b_{f}}$ & $\mathbf{N}_{\Delta t}$ & $\mathbf{N}_{\text {Chop }}$ & $\mathbf{N}_{\text {Newton }}$ & $\mathbf{N}_{\text {GMRes }}$ & CPU(s) & $\mathbf{N}_{\Delta t}$ & $\mathbf{N}_{\text {Chop }}$ & $\mathbf{N}_{\text {Newton }}$ & $\mathbf{N}_{\text {GMRes }}$ & CPU(s) \\
\hline 10 & 226 & 2 & 4.2 & 25.9 & 4638 & 226 & 2 & 4.3 & 26.2 & 5523 \\
\hline $10^{2}$ & 294 & 21 & 10.7 & 20.1 & 14557 & 246 & 8 & 7.5 & 22.2 & 9016 \\
\hline $10^{3}$ & 297 & 22 & 11.7 & 19.7 & 16183 & 225 & 1 & 5.5 & 24.2 & 6245 \\
\hline $10^{4}$ & 304 & 24 & 12.9 & 19.8 & 17742 & 225 & 1 & 4.8 & 25.1 & 5492 \\
\hline $10^{5}$ & 313 & 26 & 12.8 & 19.6 & 18346 & 235 & 4 & 5.4 & 23.9 & 6260 \\
\hline$\infty$ & $\mathrm{n} / \mathrm{a}$ & $\mathrm{n} / \mathrm{a}$ & $\mathrm{n} / \mathrm{a}$ & $\mathrm{n} / \mathrm{a}$ & $\mathrm{n} / \mathrm{a}$ & 235 & 4 & 5.3 & 23.9 & 6448 \\
\hline
\end{tabular}

Table 2: Numerical results for the pressure-saturation and variable switch formulations for $b_{m}=10^{5} \mathrm{~Pa}, p_{\text {ent }}, m=p_{\text {ent }} f=0$, and different values of the ratio $\frac{b_{m}}{b_{f}}$ (cases $C_{1}$ and $C_{2}$ ): number $N_{\Delta t}$ of successful time steps, number $N_{C h o p}$ of time step chops, number $N_{N \text { ewton }}$ of Newton iterations per successful time step, number $N_{G M R e s}$ of GMRes iterations by Newton iteration, and CPU time in seconds.

\begin{tabular}{|c|c|c|c|c|c|c|c|c|c|c|}
\hline & \multicolumn{5}{|c|}{ pressure-saturation } & \multicolumn{5}{|c|}{ variable switch } \\
\hline$\frac{b_{m}}{b_{f}}$ & $N_{\Delta t}$ & $\mathbf{N}_{\text {Chop }}$ & $\mathbf{N}_{\text {Newton }}$ & $\mathbf{N}_{\text {GMRes }}$ & CPU(s) & $N_{\Delta t}$ & $\mathbf{N}_{\text {Chop }}$ & $\mathbf{N}_{\text {Newton }}$ & $\mathbf{N}_{\text {GMRes }}$ & CPU(s) \\
\hline 2 & 221 & 0 & 3 & 29.2 & 3937 & 221 & 0 & 3.1 & 28.9 & 4479 \\
\hline 10 & 398 & 52 & 9.9 & 20.2 & 23400 & 262 & 13 & 6.8 & 22.7 & 10378 \\
\hline $10^{2}$ & $\mathrm{n} / \mathrm{c}$ & $\mathrm{n} / \mathrm{c}$ & $\mathrm{n} / \mathrm{c}$ & $\mathrm{n} / \mathrm{c}$ & $\mathrm{n} / \mathrm{c}$ & 269 & 14 & 9.9 & 20.8 & 14185 \\
\hline $10^{3}$ & $\mathrm{n} / \mathrm{c}$ & $\mathrm{n} / \mathrm{c}$ & $\mathrm{n} / \mathrm{c}$ & $\mathrm{n} / \mathrm{c}$ & $\mathrm{n} / \mathrm{c}$ & 285 & 18 & 8.9 & 20.1 & 13740 \\
\hline $10^{4}$ & $\mathrm{n} / \mathrm{c}$ & $\mathrm{n} / \mathrm{c}$ & $\mathrm{n} / \mathrm{c}$ & $\mathrm{n} / \mathrm{c}$ & $\mathrm{n} / \mathrm{c}$ & 242 & 6 & 6.9 & 22.8 & 9067 \\
\hline $10^{5}$ & $\mathrm{n} / \mathrm{c}$ & $\mathrm{n} / \mathrm{c}$ & $\mathrm{n} / \mathrm{c}$ & $\mathrm{n} / \mathrm{c}$ & $\mathrm{n} / \mathrm{c}$ & 276 & 16 & 7.5 & 21.3 & 11516 \\
\hline$\infty$ & $\mathrm{n} / \mathrm{a}$ & $\mathrm{n} / \mathrm{a}$ & $\mathrm{n} / \mathrm{a}$ & $\mathrm{n} / \mathrm{a}$ & $\mathrm{n} / \mathrm{a}$ & 299 & 22 & 8.1 & 19.1 & 10770 \\
\hline
\end{tabular}

Table 3: Numerical results for the pressure-saturation and variable switch formulations for $b_{m}=10^{5} \mathrm{~Pa}, p_{\text {ent }}, \mathrm{m}=10^{5} \mathrm{~Pa}$, $p_{e n t, f}=0$ and different values of the ratio $\frac{b_{m}}{b_{f}}$ (cases $C_{3}$ and $C_{4}$ ): number $N_{\Delta t}$ of successful time steps, number $N_{C h o p}$ of time step chops, number $N_{N e w t o n}$ of Newton iterations per successful time step, number $N_{G M R e s}$ of GMRes iterations by Newton iteration, and CPU time in seconds.

\begin{tabular}{|c|c|c|c|c|}
\hline \multicolumn{5}{|c|}{ variable switch } \\
\hline $\mathbf{N}_{\boldsymbol{\Delta} \mathbf{t}}$ & $\mathbf{N}_{\text {Chop }}$ & $\mathbf{N}_{\text {Newton }}$ & $\mathbf{N}_{\text {GMRes }}$ & CPU(s) \\
\hline 221 & 0 & 5.8 & 26.3 & 5948 \\
\hline
\end{tabular}

Table 4: Numerical results for the pressure-saturation and variable switch formulations for $b_{m}=b_{f}=0, p_{\text {ent }} m=10^{5}$ $\mathrm{Pa}$, and $p_{\text {ent }, f}=0$ (case $\left.C_{5}\right)$ : number $N_{\Delta t}$ of successful time steps, number $N_{C h o p}$ of time step chops, number $N_{N e w t o n}$ of Newton iterations per successful time step, number $N_{G M}$ Res of GMRes iterations by Newton iteration, and CPU time in seconds.

\subsection{Oil migration in a $2 \mathrm{D}$ basin}

In this section we consider the oil migration in a faulted 2D basin exhibited in Figure 19 initially saturated with water. Note that, according to our previous notations, the oil phase stands for the phase $\alpha=g$ in this test case. The domain $\Omega$ is of extension $(0,400) m \times(0,800) m$ and the fault width is assumed to be constantly $d_{f}=4 \mathrm{~m}$. The 2D triangular mesh of the domain $\Omega$ (see Figure 19 ) is extended to $3 \mathrm{D}$ by one layer of prisms since our code deals with $3 \mathrm{D}$ meshes. The characteristics of the resulting mesh are presented in table below

\begin{tabular}{|c|c|c|c|}
\hline$N b_{\text {cells }}$ & $N b_{\text {nodes }}$ & $N b_{\text {FracF }}$ & linear system d.o.f. \\
\hline 16889 & 17226 & 176 & 17284 \\
\hline
\end{tabular}

matrix. At the end of the simulation, the fractures are again fully filled with the gas phase and the water phase above the residual saturation is only partially removed during the production period due to the water retention by capillary effect. Figures 17 and 18 exhibit the instantaneous and cumulated flow rates of water and gas at the well with a positive value for production and a negative value for injection. Figures 15, 16, 17 and 18 show that the larger the difference between the capillary pressure in the matrix and in the fractures, leads to a larger amount of water which is retained in the matrix.
Initially, the reservoir is saturated with the water phase (of constant density $1000 \mathrm{~kg} / \mathrm{m}^{3}$ and viscosity $\mathrm{kg} / \mathrm{m}^{3}$ and viscosity $\left.510^{-3} \mathrm{~Pa} \cdot \mathrm{s}\right)$ is injected at the bottom boundary of the bottom fault, which is managed by imposing non-homogeneous Neumann conditions at the injection location. The oil then rises by gravity, thanks to its lower density compared to water and by the overpressure induced by the imposed injection rate. Dirichlet boundary conditions are imposed at the upper $10^{-3} \mathrm{~Pa} \cdot \mathrm{s}$ ), and the oil phase (of constant density 700 

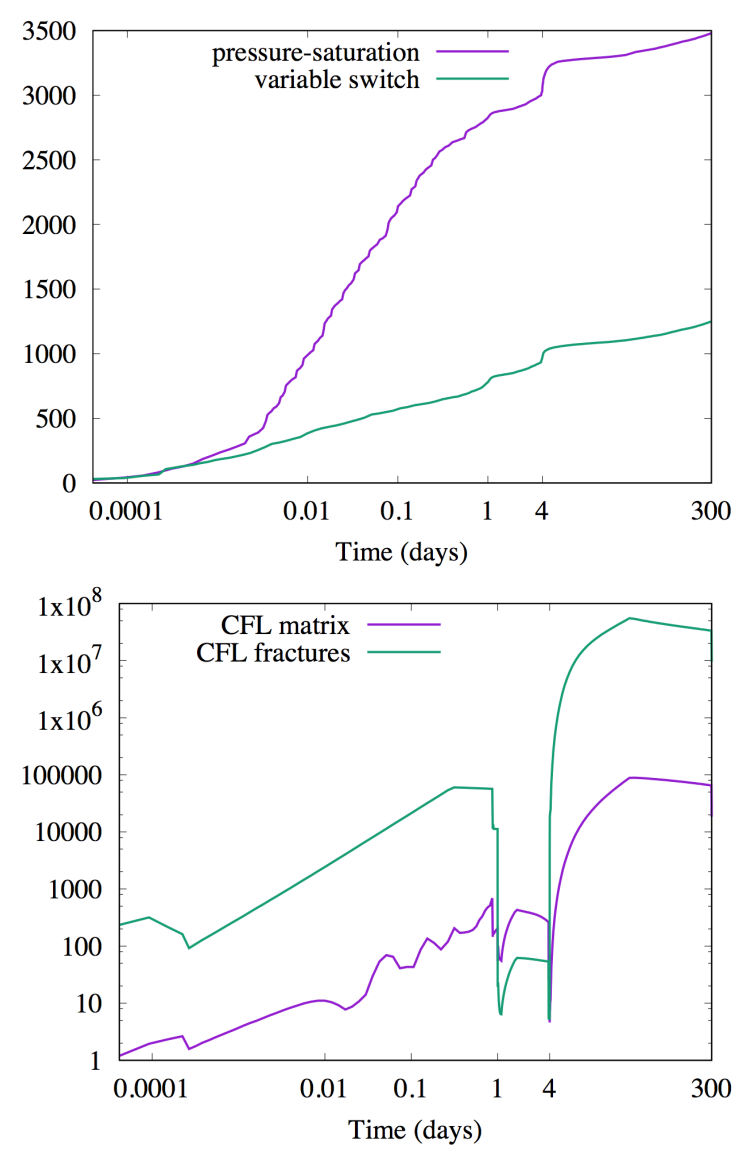

Fig. 14: For test case $C_{1}$ with $b_{m}=10^{5} \mathrm{~Pa}, p_{\text {ent, } m}=$ $p_{\text {ent }, f}=0, \frac{b_{m}}{b_{f}}=1000$ : cumulated number of Newton iterations as a function of time for the pressure-saturation and variable switch formulations (left), CFL numbers in the matrix and in the fractures as a function of time obtained with the variable switch formulation (right).

boundary of the domain, while the homogeneous Neumann boundary conditions are prescribed elsewhere.

The permeability field is isotropic and given by $\Lambda_{m}=$ $\lambda_{m}$ Id with $\lambda_{m}=10^{-13} \mathrm{~m}^{2}$, and $\Lambda_{f}=\lambda_{f}$ Id with $\lambda_{f}=$ $10^{-10} \mathrm{~m}^{2}$. The parameters of the Corey capillary pressures are fixed to $b_{m}=10^{6} \mathrm{~Pa}$ for cases $C_{1}, C_{2}, C_{3}$ and $C_{4}$, and to $p_{\text {ent }, m}=10^{6} \mathrm{~Pa}$ for cases $C_{3}, C_{4}$, and $C_{5}$. For cases $C_{1}$ and $C_{3}$ we will investigate the set of values $b_{f}=1,10,10^{4}, 10^{5} \mathrm{~Pa}$ in the faults. The porosity is equal to $\phi_{m}=0.2$ in the matrix and to $\phi_{f}=0.4$ in the faults. The mobilities are given for both the matrix and fault rocktypes $j=m, f$ by the Corey laws (17) with $s_{r, m}^{w}=s_{r, m}^{g}=0, k_{m, \max }^{w}=k_{m, \max }^{g}=1$, $n_{m}^{w}=n_{m}^{g}=2$ in the matrix, and by $s_{r, f}^{w}=s_{r, f}^{g}=0$, $k_{f, \max }^{w}=k_{f, \max }^{g}=1, n_{f}^{w}=n_{f}^{g}=1$ in the faults. The simulation is run over a period of 5400 days with an initial time step of 1 day and a maximum time step fixed to 180 days.
The numerical behaviour of the simulations for both variable switch and pressure-saturation formulations (for the cases $C_{1}$ and $C_{3}$ ), and for the variable switch formulation only (cases $C_{2}, C_{4}$ and $C_{5}$ ), is exhibited in Tables 5, 6 and 7. These tables exhibit the number of successful time steps, the number of time step chops, the number of Newton iterations by successful time steps, the number of GMRes iterations by Newton iteration, and the CPU time. Table 5 shows that, for the small values of $\frac{b m}{b f}$, the pressure-saturation formulation performs slightly better than the variable switch formulation. However the latter one turns out to be more robust w.r.t. the value of the ratio $\frac{b m}{b f}$ both in terms of number of Newton iterations and number of time step chops. The difference in the numerical behaviour between both is more striking in the case of the nonzero entry pressure $p_{\text {ent }, m}=10^{6} \mathrm{~Pa}$ (see Table 6 ). Again, in that case, the pressure-saturation formulation fails except for very small ratios $\frac{b m}{b f}$. Table 7 shows the good performance of the variable switch formulation even for the degenerate case $C_{5}$ both in terms of time step chops and Newton iterations.

Figure ?? exhibits in case $C_{1}$ for $\frac{b_{m}}{b_{f}}=10^{5}$ the cumulated number of Newton iterations as a function of time for the pressure-saturation and variable switch formulations. Most of the time step failures occur for the pressure-saturation formulation during the infill of the bottom fractures. Figure ?? also plots, on the same test case, the CFL numbers in the matrix and in the fractures as a function of time defined as in the previous test case and obtained with the variable switch formulation. It shows that the time steps used in this simulation are from 1 to 3 orders of magnitude larger than the ones obtained with an IMPES scheme in the fractures.

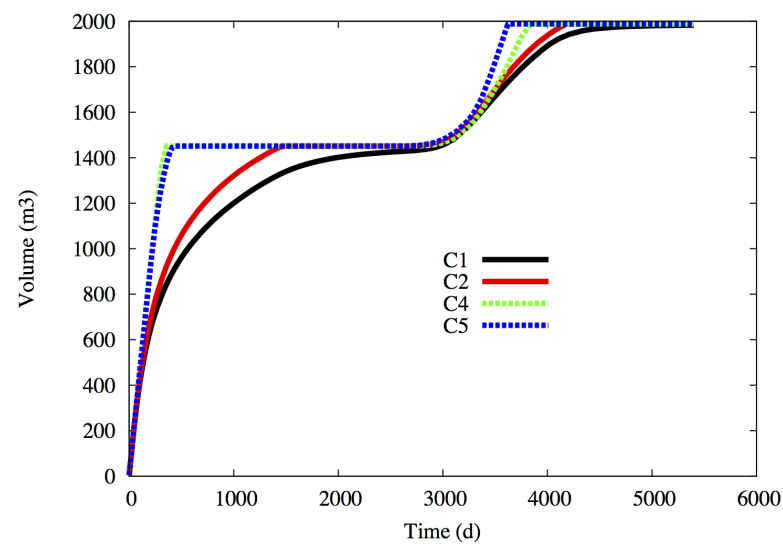

Fig. 20: Amount of oil in the faults in $\mathrm{m}^{3}$ as a function of time for the following four test cases. $C_{1}: b_{m}=10^{6} \mathrm{~Pa}, b_{f}=10^{5}$ $\mathrm{Pa}, p_{\text {ent }, m}=p_{\text {ent }, f}=0, C_{2}: b_{m}=10^{6} \mathrm{~Pa}, b_{f}=0, p_{\text {ent }, m}=$ $p_{\text {ent }, f}=0, C_{4}: b_{m}=10^{6} \mathrm{~Pa}, b_{f}=0, p_{\text {ent }, m}=10^{6} \mathrm{~Pa}$, $p_{\text {ent }, f}=0, C_{5}: b_{m}=b_{f}=0, p_{\text {ent }, m}=10^{6} \mathrm{~Pa}, p_{\text {ent }, f}=0$. 

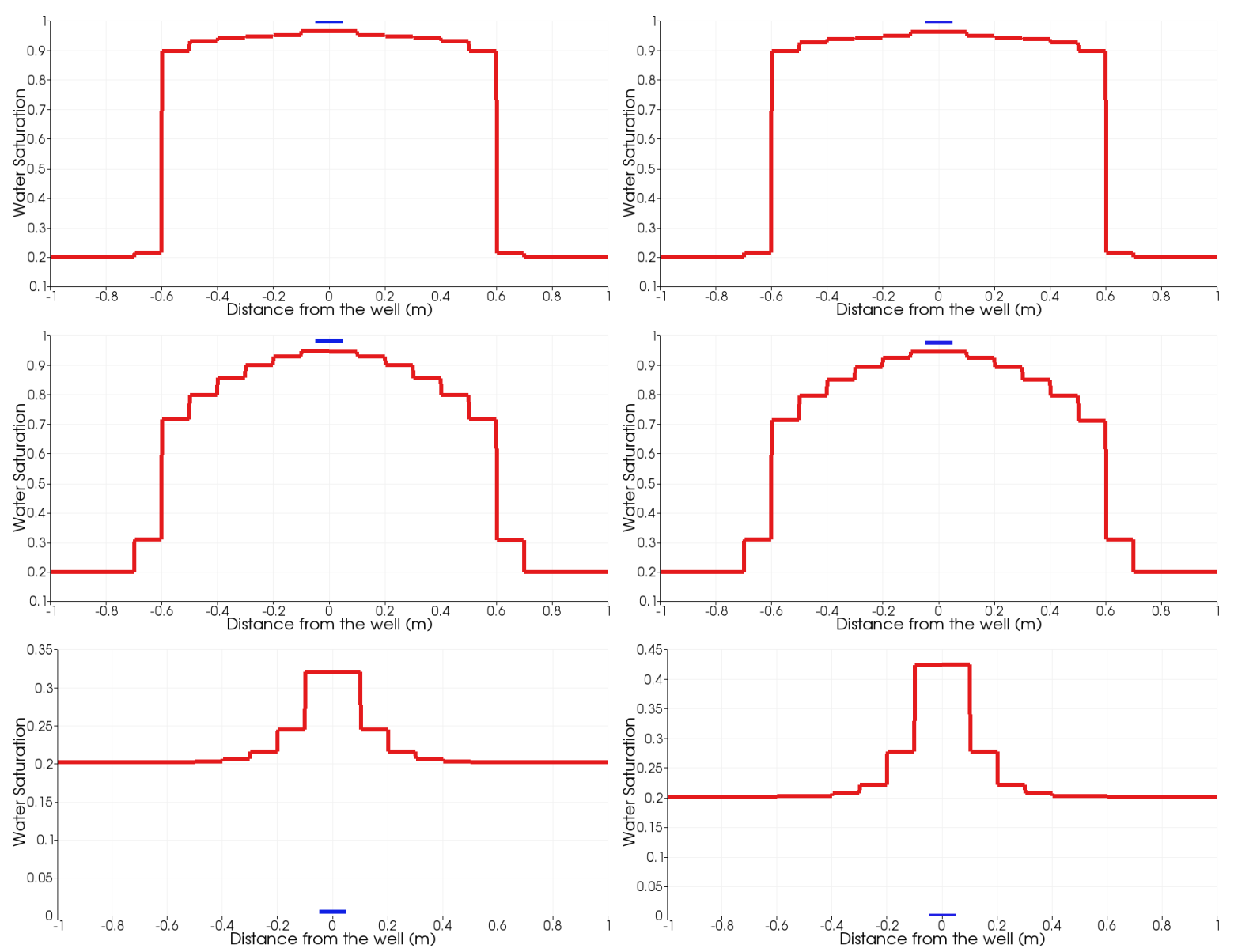

Fig. 15: Water saturation in the perforated face in the fracture at $\mathbf{x}=0$ (blue) and cut of the water saturation in the matrix (red) along the line $y=z=0$ as a function of the distance to the fracture at the end of each simulation period $t=1$ day (top), $t=4$ days (middle) and $t=300$ days (bottom). The left column correspond to $b_{m}=10^{5} \mathrm{~Pa}, b_{f}=10^{4} \mathrm{~Pa}$, $p_{\text {ent }, m}=p_{\text {ent }, f}=0$, and the right column to $b_{m}=10^{5} \mathrm{~Pa}, b_{f}=0, p_{\text {ent }, m}=p_{\text {ent }, f}=0$

Figure 20 exhibits the volume of oil in the faults as a function of time for $C_{1}, C_{2}, C_{4}$ and $C_{5}$ test case solutions obtained with the variable switch formulation. As expected, larger constrasts of capillary pressures between the matrix and the faults result in a larger amount of oil in the fault up to the total pore volume and a quicker infill. Figure 22 shows the propagation of the oil saturation in the basin at times $t=$ $360,2880,5400$ days for the $C_{1}$ case with $\frac{b m}{b f}=10$ obtained with the variable switch formulation. 
(a)
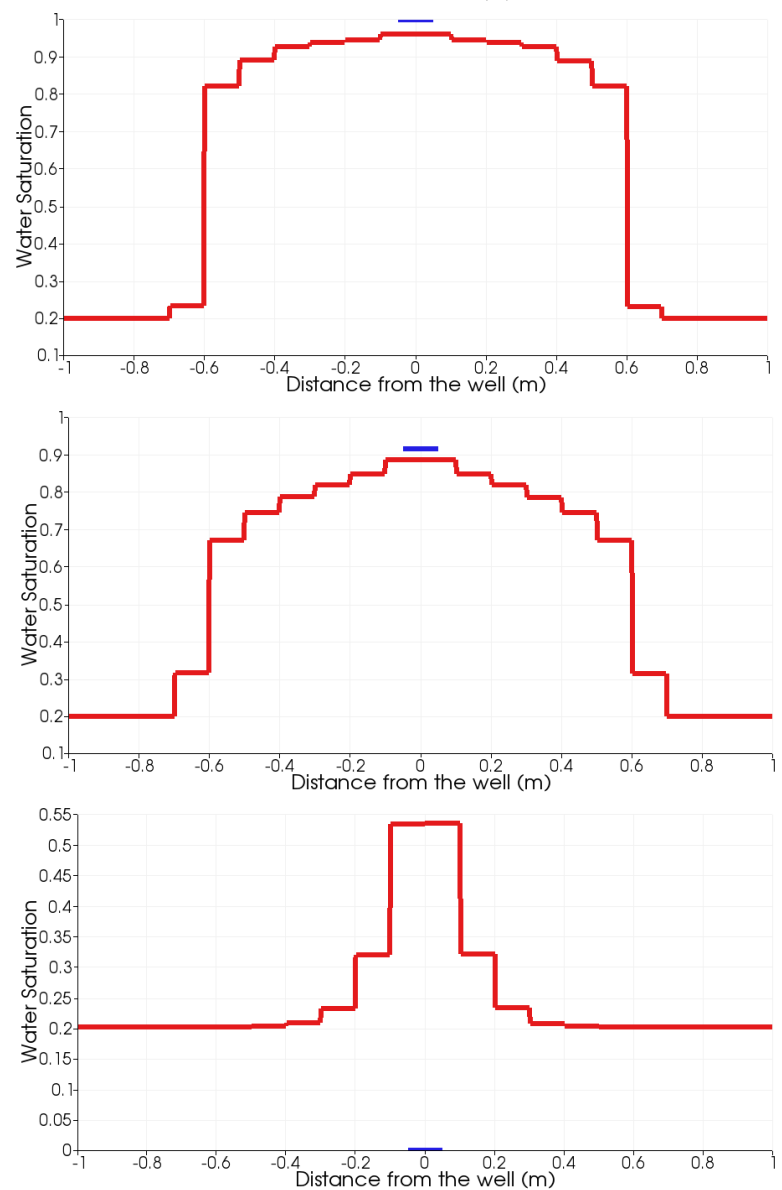

(b)
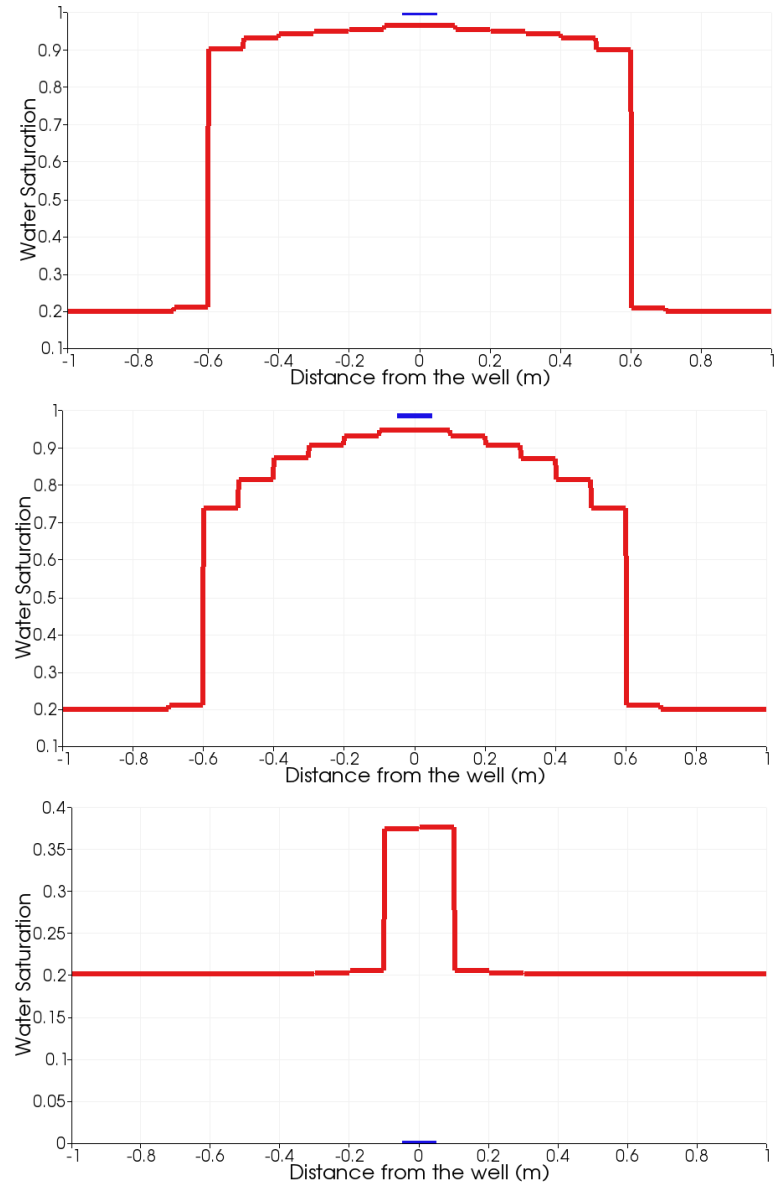

Fig. 16: Water saturation in the perforated face in the fracture at $\mathbf{x}=0$ (blue) and cut of the water saturation in the matrix (red) along the line $y=z=0$ as a function of the distance to the fracture at the end of each simulation period $t=1$ day (top), $t=4$ days (middle) and $t=300$ days (bottom). The left column correspond to $b_{m}=10^{5} \mathrm{~Pa}, b_{f}=0, p_{\text {ent }}, m=10^{5} \mathrm{~Pa}$, $p_{\text {ent }, f}=0$, and the right column to $b_{m}=b_{f}=0, p_{\text {ent }, m}=10^{5} \mathrm{~Pa}, p_{\text {ent }, f}=0$.

\begin{tabular}{|c|c|c|c|c|c|c|c|c|c|c|}
\hline & \multicolumn{5}{|c|}{ pressure-saturation } & \multicolumn{5}{|c|}{ variable switch } \\
\hline$\frac{b_{m}}{b_{f}}$ & $N_{\Delta t}$ & $\mathbf{N}_{\text {Chop }}$ & $\mathbf{N}_{\text {Newton }}$ & $\mathbf{N}_{\text {GMRes }}$ & CPU(s) & $\mathbf{N}_{\Delta t}$ & $\mathbf{N}_{\text {Chop }}$ & $\mathbf{N}_{\text {Newton }}$ & $\mathbf{N}_{\text {GMRes }}$ & CPU(s) \\
\hline 10 & 95 & 0 & 4.99 & 38.96 & 554 & 95 & 0 & 5.23 & 37.12 & 602 \\
\hline 100 & 95 & 0 & 5.78 & 23.15 & 524 & 95 & 0 & 7.29 & 22.89 & 693 \\
\hline $10^{5}$ & 364 & 72 & 15.72 & 14.52 & 3791 & 110 & 4 & 13.47 & 19.26 & 1565 \\
\hline $10^{6}$ & 304 & 57 & 15.52 & 13.47 & 3311 & 110 & 4 & 13.41 & 19.23 & 1397 \\
\hline$\infty$ & $\mathrm{n} / \mathrm{a}$ & $\mathrm{n} / \mathrm{a}$ & $\mathrm{n} / \mathrm{a}$ & $\mathrm{n} / \mathrm{a}$ & $\mathrm{n} / \mathrm{a}$ & 102 & 2 & 12.74 & 20.79 & 1188 \\
\hline
\end{tabular}

Table 5: Numerical results for the pressure-saturation, and variable switch formulations for $b_{m}=10^{6} \mathrm{~Pa}, p_{\text {ent }, m}=p_{\text {ent }, f}=0$, and different values of the ratio $\frac{b_{m}}{b_{f}}$ (cases $C_{1}$ and $C_{2}$ ): number $N_{\Delta t}$ of successful time steps, number $N_{C h o p}$ of time step chops, number $N_{N e w t o n}$ of Newton iterations per successful time step, number $N_{G M R e s}$ of GMRes iterations by Newton iteration, and CPU time in seconds. 


\begin{tabular}{|c|c|c|c|c|c|c|c|c|c|c|}
\hline & \multicolumn{5}{|c|}{ pressure-saturation } & \multicolumn{5}{|c|}{ variable switch } \\
\hline$\frac{b_{m}}{b_{f}}$ & $\mathbf{N}_{\Delta t}$ & $\mathbf{N}_{\text {Chop }}$ & $\mathbf{N}_{\text {Newton }}$ & $\mathbf{N}_{\text {GMRes }}$ & CPU(s) & $\mathbf{N}_{\Delta t}$ & $\mathbf{N}_{\text {Chop }}$ & $\mathbf{N}_{\text {Newton }}$ & $\mathbf{N}_{\text {GMRes }}$ & CPU(s) \\
\hline 10 & 102 & 2 & 6.07 & 30.67 & 707 & 95 & 0 & 5.62 & 28.49 & 572 \\
\hline 100 & $\mathrm{n} / \mathrm{c}$ & $\mathrm{n} / \mathrm{c}$ & $\mathrm{n} / \mathrm{c}$ & $\mathrm{n} / \mathrm{c}$ & $\mathrm{n} / \mathrm{c}$ & 95 & 0 & 7.38 & 26.37 & 727 \\
\hline $10^{5}$ & $\mathrm{n} / \mathrm{c}$ & $\mathrm{n} / \mathrm{c}$ & $\mathrm{n} / \mathrm{c}$ & $\mathrm{n} / \mathrm{c}$ & $\mathrm{n} / \mathrm{c}$ & 121 & 7 & 12.76 & 23.89 & 1633 \\
\hline $10^{6}$ & $\mathrm{n} / \mathrm{c}$ & $\mathrm{n} / \mathrm{c}$ & $\mathrm{n} / \mathrm{c}$ & $\mathrm{n} / \mathrm{c}$ & $\mathrm{n} / \mathrm{c}$ & 106 & 3 & 11.81 & 26.31 & 1277 \\
\hline$\infty$ & $\mathrm{n} / \mathrm{a}$ & $\mathrm{n} / \mathrm{a}$ & $\mathrm{n} / \mathrm{a}$ & $\mathrm{n} / \mathrm{a}$ & $\mathrm{n} / \mathrm{a}$ & 114 & 5 & 12.46 & 22.65 & 1321 \\
\hline
\end{tabular}

Table 6: Numerical results for the pressure-saturation and variable switch formulations for $b_{m}=10^{6} \mathrm{~Pa}, p_{\text {ent,m }}=10^{6} \mathrm{~Pa}$, $p_{e n t, f}=0$ and different values of the ratio $\frac{b_{m}}{b_{f}}$ (cases $C_{3}$ and $C_{4}$ ): number $N_{\Delta t}$ of successful time steps, number $N_{C h o p}$ of time step chops, number $N_{N e w t o n}$ of Newton iterations per successful time step, number $N_{G M R e s}$ of GMRes iterations by Newton iteration, and CPU time in seconds. 

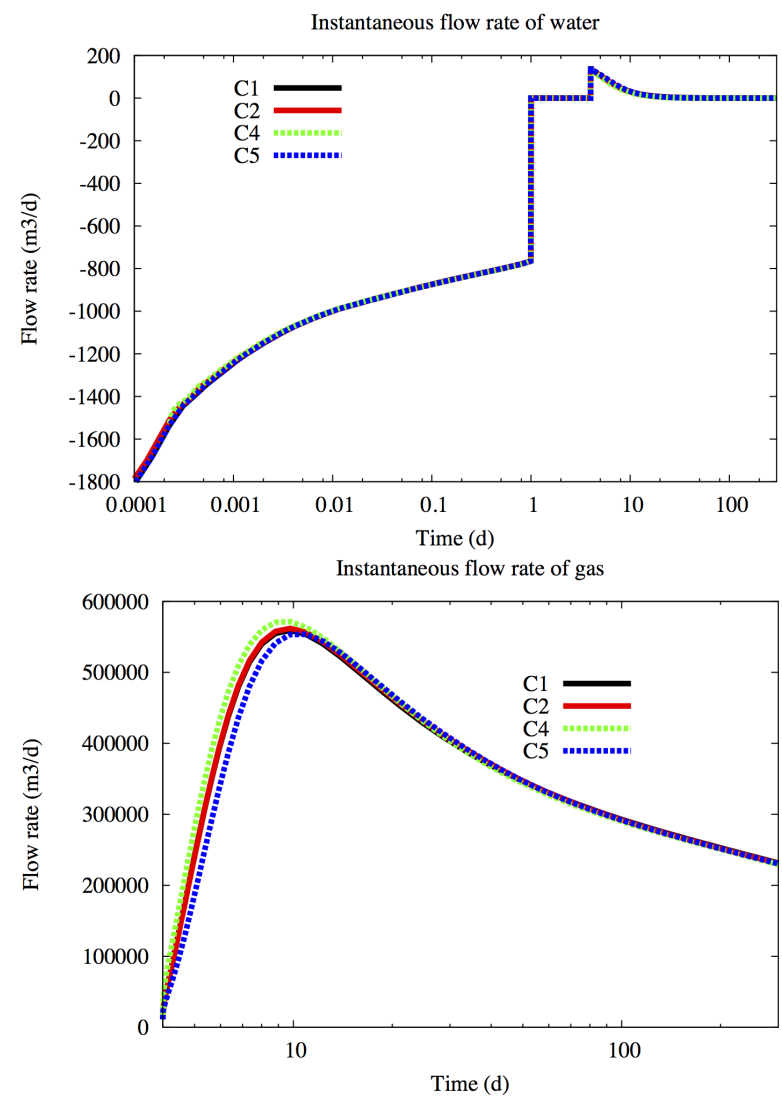

Fig. 17: Instantaneous flow rates of water (top) and of gas (bottom) in $\mathrm{m}^{3} /$ day as a function of time.

\begin{tabular}{|c|c|c|c|c|}
\hline \multicolumn{5}{|c|}{ variable switch } \\
\hline $\mathbf{N}_{\boldsymbol{\Delta} \mathbf{t}}$ & $\mathbf{N}_{\text {Chop }}$ & $\mathbf{N}_{\text {Newton }}$ & $\mathbf{N}_{\text {GMRes }}$ & CPU(s) \\
\hline 185 & 17 & 14.28 & 25.92 & 2147 \\
\hline
\end{tabular}

Table 7: Numerical results for the pressure-saturation and variable switch formulations for $b_{m}=b_{f}=0, p_{\text {ent }, m}=10^{6}$ $\mathrm{Pa}$, and $p_{\text {ent }, f}=0$ (case $C_{5}$ ): number $N_{\Delta t}$ of successful time steps, number $N_{C h o p}$ of time step chops, number $N_{N e w t o n}$ of Newton iterations per successful time step, number $N_{G M}$ Res of GMRes iterations by Newton iteration, and CPU time in seconds.
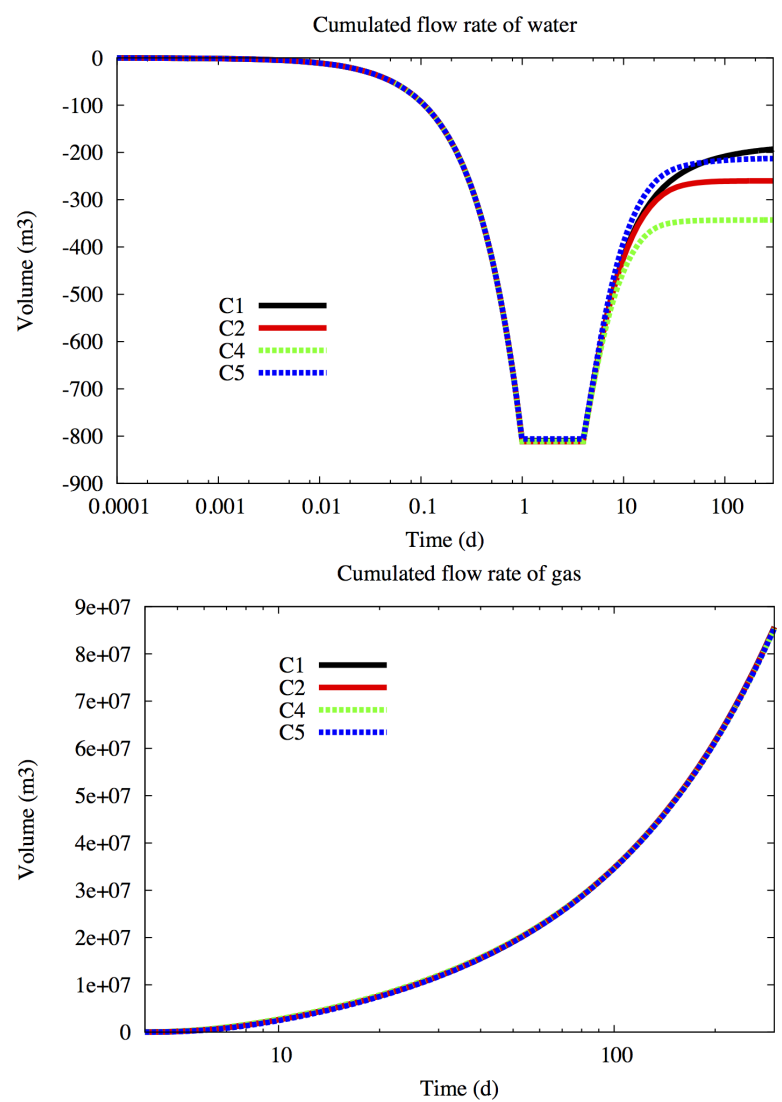

Fig. 18: Cumulated flow rates in $\mathrm{m}^{3}$ of water (top) and gas (bottom) as a function of time.
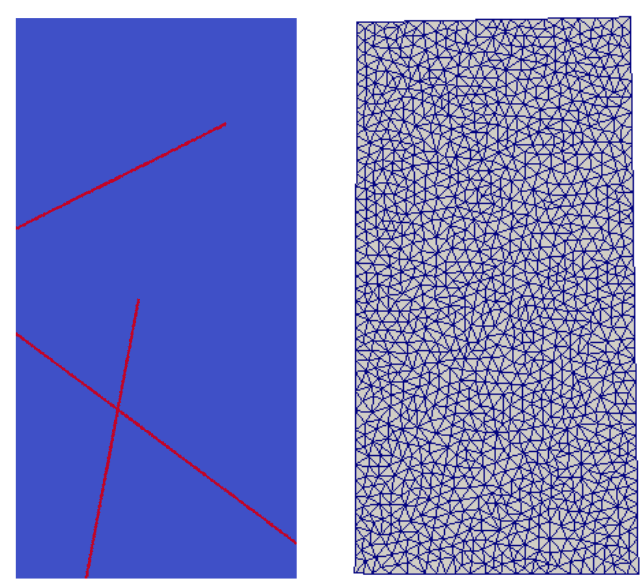

Fig. 19: On the left: geometry of the basin $\Omega=(0,400) m \times$ $(0,800) m$ with the fault network in red and the matrix domain in blue. On the right: coarse triangular mesh (with 2441 cells) of the $2 \mathrm{D}$ basin conforming to the fault network. 

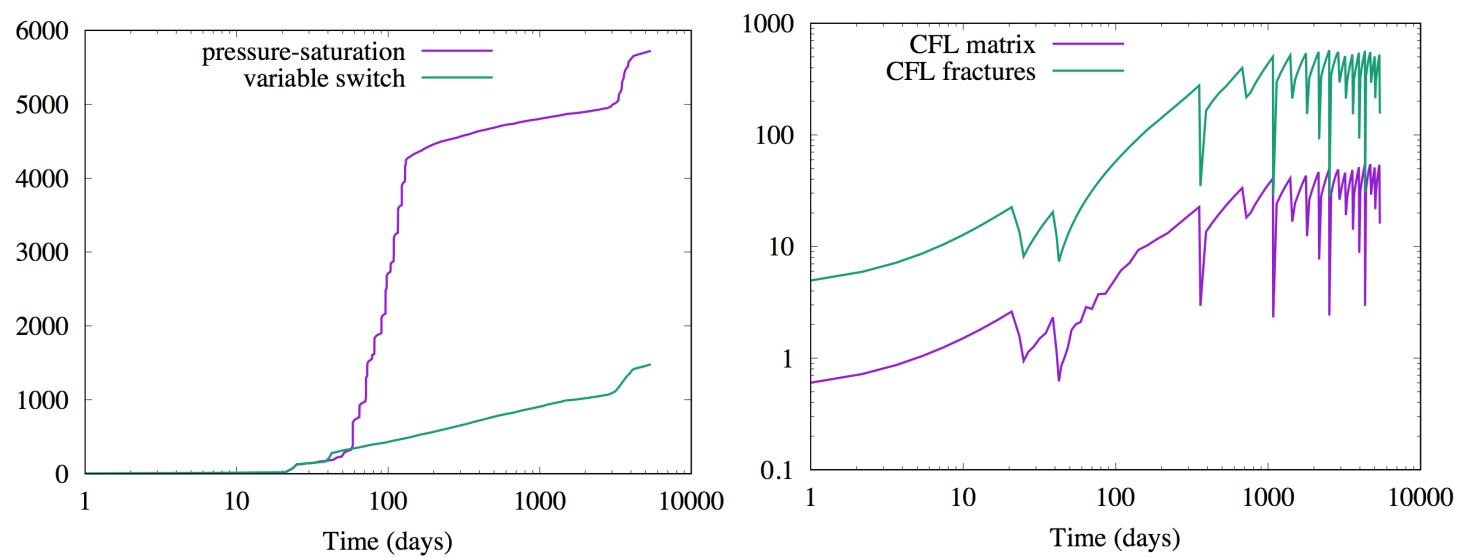

Fig. 21: For test case $C_{1}$ with $b_{m}=10^{6} \mathrm{~Pa}, p_{\text {ent }, m}=p_{\text {ent }, f}=0, \frac{b_{m}}{b_{f}}=10^{5}$ : cumulated number of Newton iterations as a function of time for the pressure-saturation and variable switch formulations (left), CFL in the matrix and in the fractures as a function of time obtained with the variable switch formulation (right).
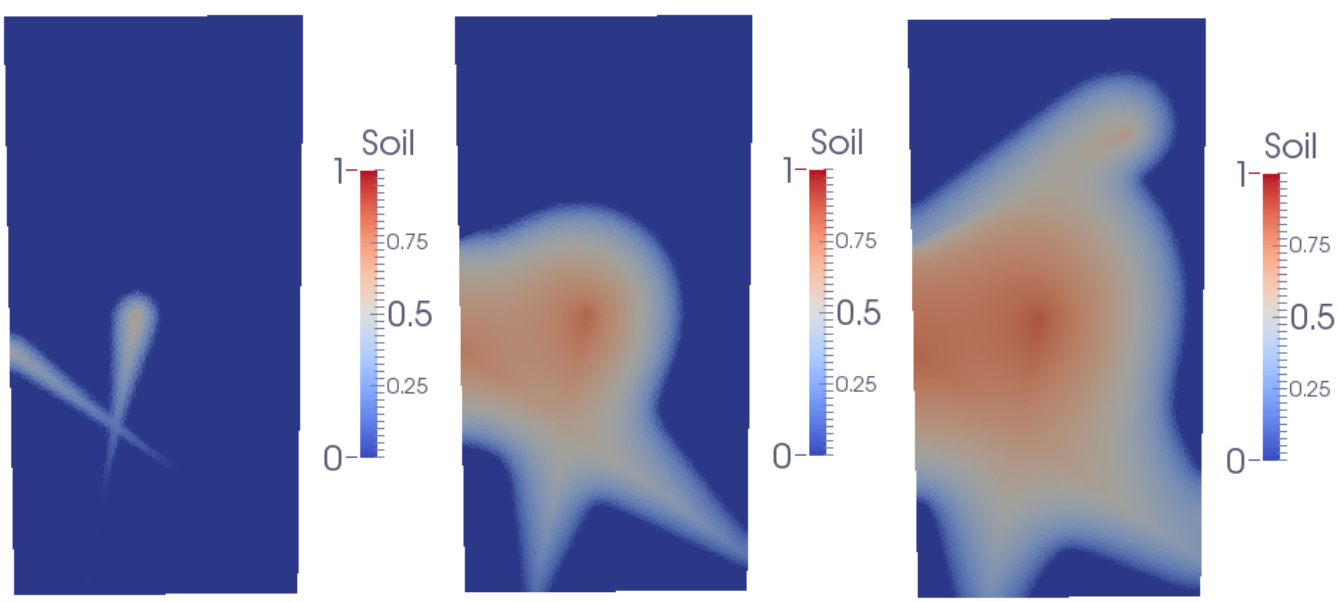

Fig. 22: Oil saturation at $t=360$ days (left), $t=2880$ days (middle) and $t=5400$ at the end (right) of simulation obtained for the $C_{1}$ case with $\frac{b m}{b f}=10$ and the variable switch formulation. 


\section{Acknowledgements}

The authors would like to thank ENGIE EP and Storengy for supporting this work and allowing its publication. This work was also supported by the GeoPor project funded by the French National Research Agency (ANR) with the grant ANR-13-JS01-0007-01 (project GEOPOR).

\section{References}

1. Ahmed, R., Edwards, M., Lamine, S., Huisman, B.: Control-volume distributed multi-point flux approximation coupled with a lower-dimensional fracture model. Journal of Computational Physics 284, 462-489 (2015)

2. Alboin, C., Jaffre, J., Roberts, J., C., S.: Modeling fractures as interfaces for flow and transport in porous media. Fluid flow and transport in porous media 295, 13-24 (2002)

3. Angot, P., Boyer, F., Hubert, F.: Asymptotic and numerical modelling of flows in fractured porous media. Mathematical Modelling and Numerical Analysis 43, 239-275 (2009)

4. Brenner, K., Groza, M., Guichard, C., Lebeau, G., Masson, R.: Gradient discretization of hybrid dimensional darcy flows in fractured porous media. Numerische Mathematik pp. 1-41 (2015)

5. Brenner, K., Groza, M., Guichard, C., Masson, R.: Vertex approximate gradient scheme for hybrid dimensional two-phase darcy flows in fractured porous media. Mathematical Modelling and Numerical Analysis 49, 303-330 (2015)

6. Brenner, K., Hennicker, J., Masson, R., Samier, P.: Gradient discretization of hybrid-dimensional darcy flow in fractured porous media with discontinuous pressures at matrix-fracture interfaces. IMA Journal of Numerical Analysis (2016)

7. Brenner, K., Hennicker, J., Masson, R., Samier, P.: Hybrid dimensional modelling and discretization of two phase darcy flow through dfn in porous media. In: ECMOR XV- 15th European Conference on the Mathematics of Oil Recovery, 29 august-1 september 2016, Amsterdam, Netherlands (2016)

8. Cancès, C., Pierre, M.: An existence result for multidimensional immiscible two-phase flows with discontinuous capillary pressure field. SIAM J. Math. Anal. 44, 966-992 (2012)

9. Ding, D., Langouet, H., Jeannin, L.: Simulation of fracturing induced formation damage and gas production from fractured wells in tight gas reservoirs. SPE $\mathbf{1 5 3 2 5 5}$ (2012)

10. Eymard, R., Guichard, C., Herbin, R.: Small-stencil 3d schemes for diffusive flows in porous media. Mathematical Modelling and Numerical Analysis 46, 265-290 (2010)

11. Eymard, R., Guichard, C., Herbin, R., Masson, R.: Gradient schemes for two-phase flow in heterogeneous porous media and richards equation. ZAMM - Journal of Applied Mathematics and Mechanics 94, 560-585 (2014)

12. Flauraud, E., Nataf, F., Faille, I., Masson, R.: Domain decomposition for an asymptotic geological fault modeling. Comptes Rendus à l'académie des Sciences, Mécanique 331, 849-855 (2003)
13. Hoteit, J., Firoozabadi, A.: An efficient numerical model for incompressible two-phase flow in fracture media. Advances in Water Resources 31, 891-905 (2008)

14. Karimi-Fard, M., Durlofsky, L., Aziz, K.: An efficient discrete-fracture model applicable for general-purpose reservoir simulators. SPE Journal (2004)

15. Martin, V., Jaffré, J., Roberts, J.: Modeling fractures and barriers as interfaces for flow in porous media. SIAM J. Sci. Comput. 26, 1667-1691 (2005)

16. Monteagudu, J., Firoozabadi, A.: Control-volume model for simulation of water injection in fractured media: incorporating matrix heterogeneity and reservoir wettability effects. SPE Journal 12, 355-366 (2007)

17. Reichenberger, V., Jakobs, H., Bastian, P., Helmig, R.: A mixed-dimensional finite volume method for multiphase flow in fractured porous media. Adv. Water Resources 29, 1020-1036 (2006)

18. Sandve, T., Berre, I., Nordbotten, J.: An efficient multipoint flux approximation method for discrete fracturematrix simulations. Journal of Computational Physics 231, 3784-3800 (2012)

19. Si, H.: http://tetgen.org (2011)

20. Tunc, X., Faille, I., Gallouët, T., Cacas, M., Havé, P.: A model for conductive faults with non matching grids. Comp. Geosciences 16, 277-296 (2012) 